Florida International University FIU Digital Commons

$11-14-2012$

\title{
Improved Electronics for the Hall A Detectors at JLab: Summing Modules and VDC Amplifier/ Discriminator Cards
}

Casey M. Neville

Florida International University, cnevi001@fiu.edu

DOI: $10.25148 /$ etd.FI12120702

Follow this and additional works at: https://digitalcommons.fiu.edu/etd

\section{Recommended Citation}

Neville, Casey M., "Improved Electronics for the Hall A Detectors at JLab: Summing Modules and VDC Amplifier/Discriminator Cards" (2012). FIU Electronic Theses and Dissertations. 794.

https://digitalcommons.fiu.edu/etd/794 


\title{
FLORIDA INTERNATIONAL UNIVERSITY
}

Miami, Florida

\section{IMPROVED ELECTRONICS FOR THE HALL A DETECTORS AT JLAB: SUMMING MODULES AND VDC AMPLIFIER/DISCRIMINATOR CARDS}

\author{
A thesis submitted in partial fulfillment of the \\ requirements for the degree of \\ MASTER OF SCIENCE \\ in \\ PHYSICS \\ by
}

Casey Michael Neville 
To: Dean Kenneth G. Furton

College of Arts and Sciences

This thesis, written by Casey Michael Neville, and entitled Improved Electronics for the Hall A Detectors at JLab: Summing Modules and VDC Amplifier/Discriminator Cards having been approved in respect to style and intellectual content, is referred to you for judgment.

We have read this thesis and recommend that it be approved.

$\begin{array}{r}\hline \text { Laird Kramer } \\ \hline \text { Joerg Reinhold } \\ \hline \text { Pete Markowitz, Major Professor }\end{array}$

Date of Defense: November 14, 2012

The thesis of Casey Michael Neville is approved.

Dean Kenneth G. Furton

College of Arts and Sciences

Dean Lakshmi N. Reddi

University Graduate School

Florida International University, 2012 


\section{DEDICATION}

I would like to dedicate this thesis to my fiancé Manuela Noriega, without her support and encouragement completion of the graduate thesis and coursework would not have been possible. 


\section{ACKNOWLEDGMENTS}

I would like to acknowledge the patience, guidance, and continued support during both my undergraduate and graduate studies from my committee: Dr. Laird Kramer for furthering my career by employing me in the CHEPREO program, Dr. Joerg Reinhold for inviting me to my first trip to JLab for my summer 2010 research in the FAST program, and Dr. Pete Markowitz for having understanding and support during both my undergraduate and graduate research. My academic career would not have advanced to this point without each of your support. Thank you. 


\begin{abstract}
OF THE THESIS
IMPROVED ELECTRONICS FOR THE HALL A DETECTORS AT JLAB: SUMMING MODULES AND VDC AMPLIFIER/DISCRIMINATOR CARDS

by

Casey Michael Neville
\end{abstract}

Florida International University, 2012

Miami, Florida

Professor Pete Markowitz, Major Professor

Testing of summing electronics and VDC A/D Cards was performed to assure proper functioning and operation within defined parameters. In both the summing modules and the VDC A/D cards, testing for minimum threshold voltage for each channel and crosstalk between neighboring channels was performed. Additionally, the modules were installed in Hall A with input signals from shower detectors arranged to establish a trigger by summing signals together with the use of tested modules. Testing involved utilizing a pulser to mimic PMT signals, a discriminator, an attenuator, a scaler, a level translator, an oscilloscope, a high voltage power supply, and a special apparatus used to power and send signal to the A/D cards. After testing, modules were obtained that meet necessary criteria for use in the APEX experiment, and the A/D cards obtained were determined to have adequate specifications for their utilization, with specific results included in the appendix. 


\section{TABLE OF CONTENTS}

CHAPTER

PAGE

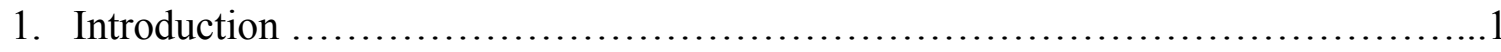

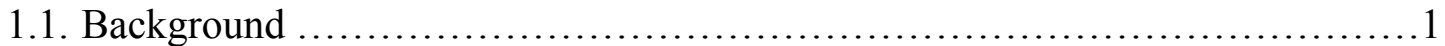

1.1.1. The Standard Model and The Dark Matter Problem .......................

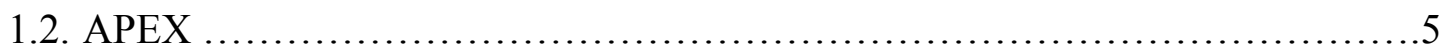

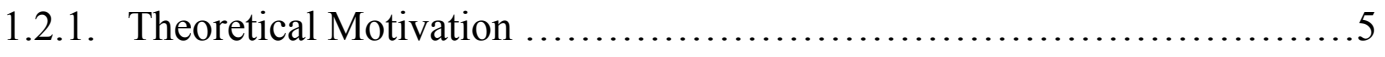

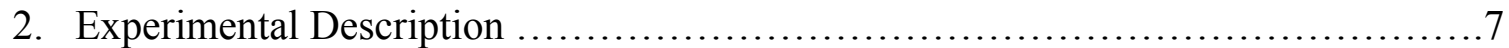

2.1. Electron Beam ....................................................

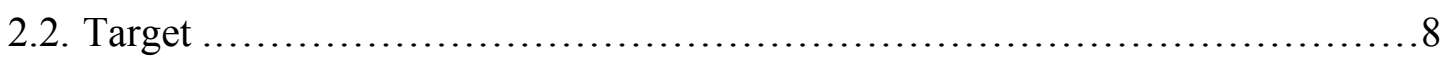

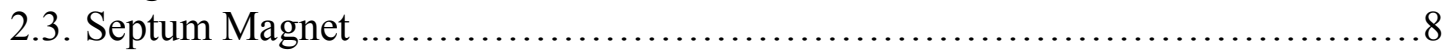

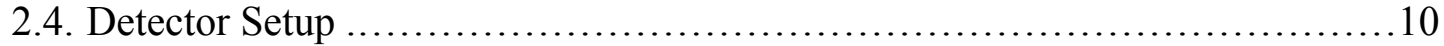

2.4.1. Vertical Drift Chambers (VDCs) ..................................11

2.4.2. Shower Counters .............................................. 14

3. Research Focus ..................................................... 15

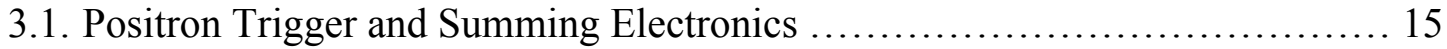

3.2. Momentum Reconstruction: VDC Electronics............................. 19

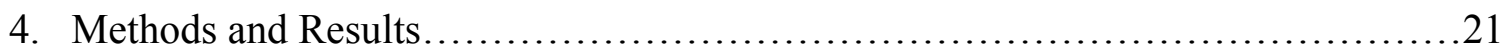

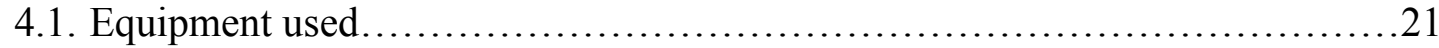

4.1.1. Pulser............................................................. 21

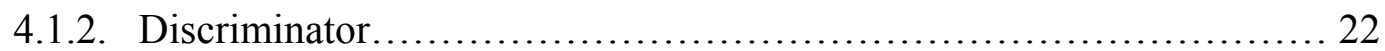

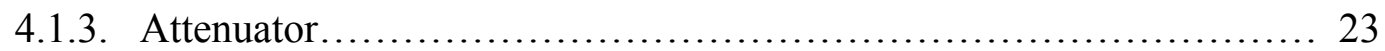

4.1.4. Level Translator............................................ 24

4.2. Testing of Summing Electronics....................................... 25

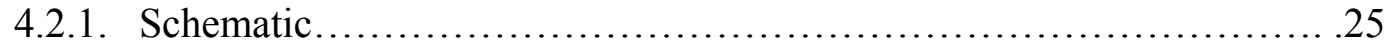

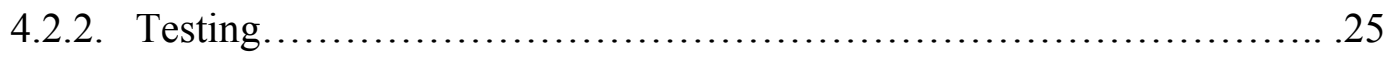

4.2.3. Taking Measurements from the Summing Modules................... 26

4.3. VDC Electronics................................................... 27

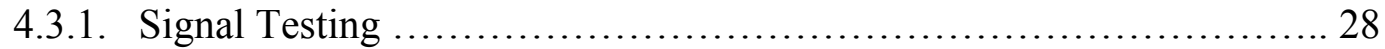

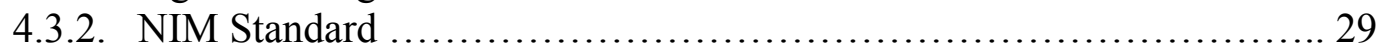

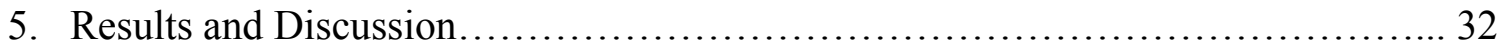

5.1. VDC A/D Card results ............................................... 32

5.2. Summation module results .............................................. 32

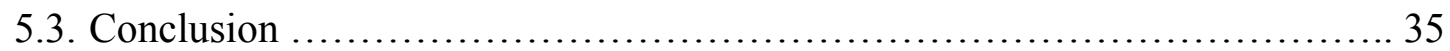

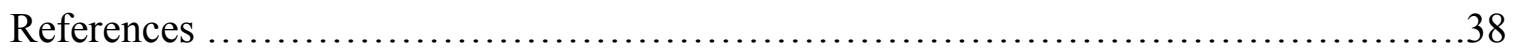

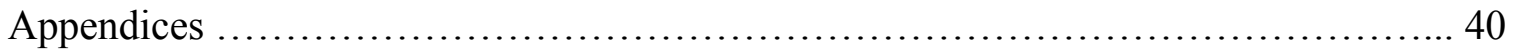




\section{LIST OF FIGURES}

FIGURE

PAGE

1. Plot of stellar velocity vs. distance from galactic center $\ldots \ldots \ldots \ldots \ldots \ldots \ldots . \ldots 2$

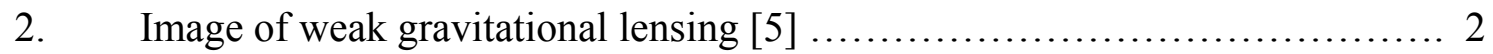

3. Geometric depiction of the mechanism of gravitational lensing ............. 4

4. Schematic of Thomas Jefferson accelerator and Halls [9] $\ldots \ldots \ldots \ldots \ldots \ldots \ldots . \ldots$

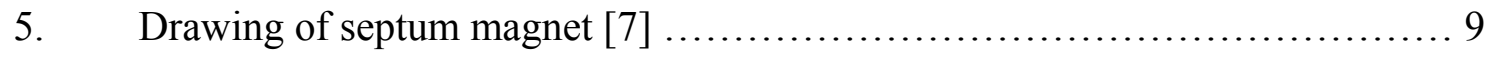

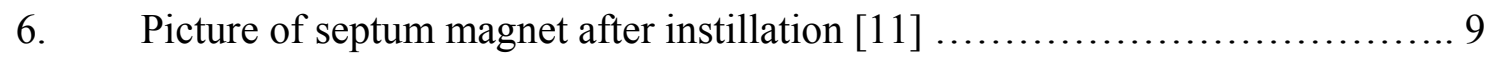

7. Drawing of detector package in right arm of HRS in Hall A [9] ...............10

8. Picture of vertical drift chamber before installation .................... 12

9. Figure depicting VDC layout after instillation and particle trajectory ....... 13

10. Diagram of front plate of summation module ............................ 16

11. Diagram of arrangement of installed summation modules ...................16

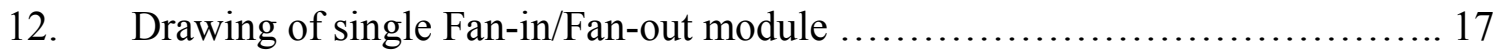

13. Schematic of trigger logic and arrangement of signals in Fan-in/Fan-out ....... 17

14. Picture of arrangement of summing modules after installation $\ldots \ldots \ldots \ldots \ldots \ldots 18$

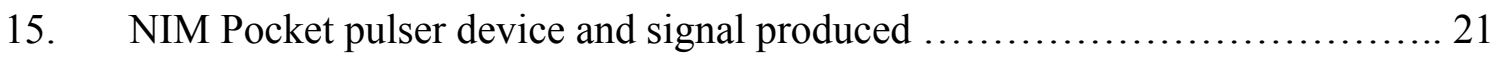

16. Picture of pulser signal after discriminator ............................... 22

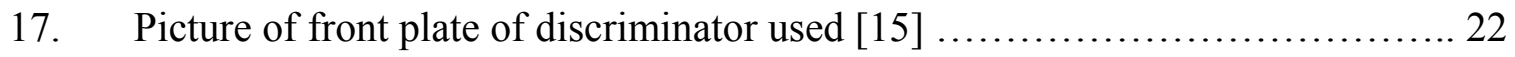

18. Picture of attenuator connected for use in testing summation modules .......... 23

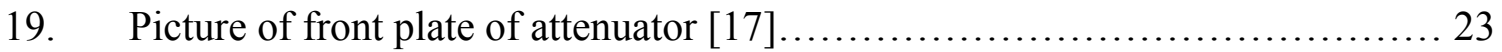

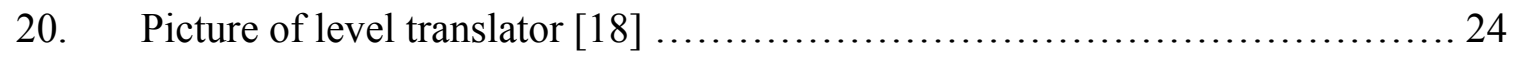

21. Picture of apparatus for testing summation modules $\ldots \ldots \ldots \ldots \ldots \ldots \ldots \ldots \ldots . .25$ 
22. Diagram depicting path of signal through testing apparatus .................. 25

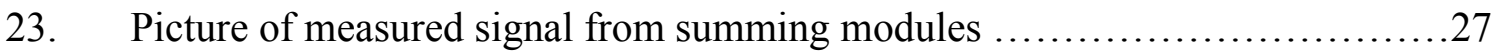

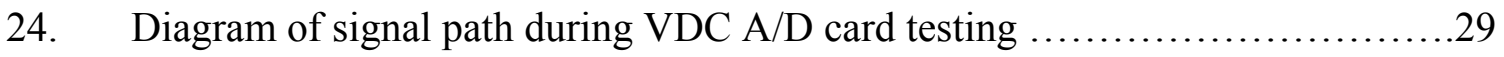

25. Frequency distribution for VDC A/D card threshold voltage ............................33

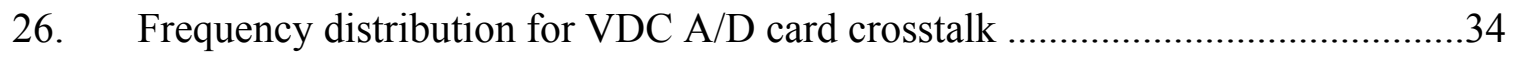

27. Frequency distribution of summing module threshold voltage ..........................36 


\section{Introduction}

\subsection{Background}

\subsubsection{The Standard Model and The Dark Matter Problem}

For years, scientists have been attempting to explain several phenomena that fail to adhere to contemporary scientific models and elude fundamental understanding [1]. These observations, most of which are astronomical, have resulted in enormous theoretical activity, the most notable of which have given rise to the theory of Dark Matter [2]. This theory states that throughout the universe there exists a material that under normal circumstances does not interact with matter except through a gravitational attraction. In general, it will not interact through the electromagnetic spectrum, which makes it difficult to detect through conventional methods. Matter is detected by observing the various interactions that take place between particles through the electromagnetic force; a property that by definition, dark matter is unable to accomplish [2]. However, theory predicts that dark matter should weakly interact with normal matter through the exchange of the $A$ ' virtual photon. Evidence for the existence of Dark Matter and its gravitational interaction with visible matter appears in the following forms.

Much of the evidence for dark matter appears in the observation of galaxies and galaxy clusters. Observations of galaxy clusters show that the velocities at which they orbit within the cluster cannot be achieved utilizing only the gravitational force available from the observable matter [Figure 1]. The same applies to stars orbiting a galaxy; the stars that orbit near the edge of the galaxy should orbit much slower than stars near the center, but often they do not [3]. Not show in Figure 1 the projected (theoretical) 
velocities of objects at the corresponding distances, but from basic centripetal acceleration principles it is understood that as distance from the center increases, and with no increase in the force applied, velocity will decrease. This extra velocity, in both stars and galaxies around a cluster, appears to be the result of dark matter that has a significant gravitational interaction with these orbiting stars and galaxies.

Evidence of dark matter also comes in the form of observed gravitational lensing; a process in which light is bent around an object by its powerful gravitational field [4].

Observing an object like a galaxy that is undergoing gravitational lensing appears to warp

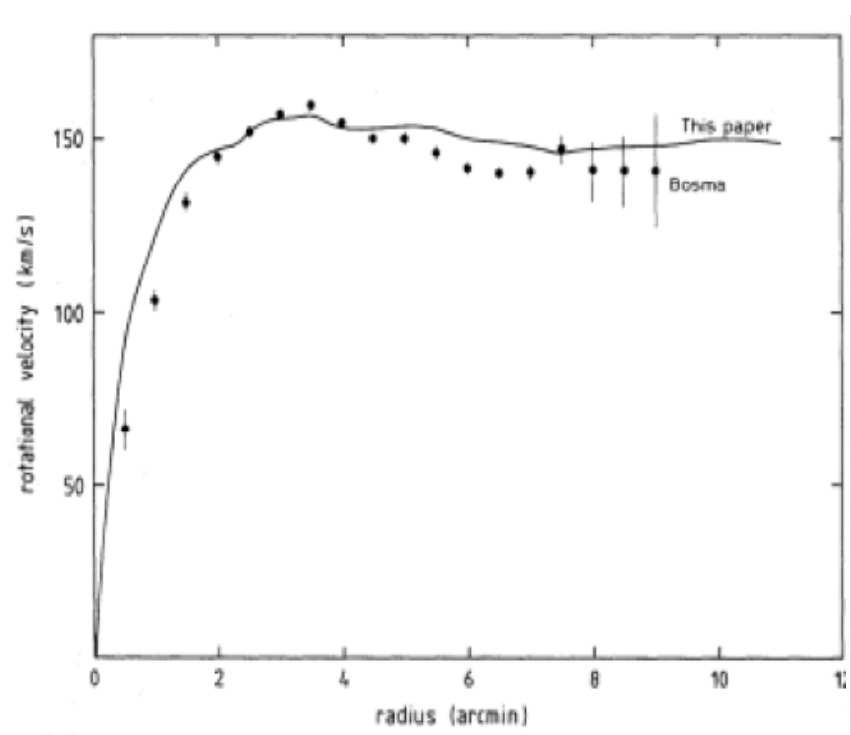

Figure 1

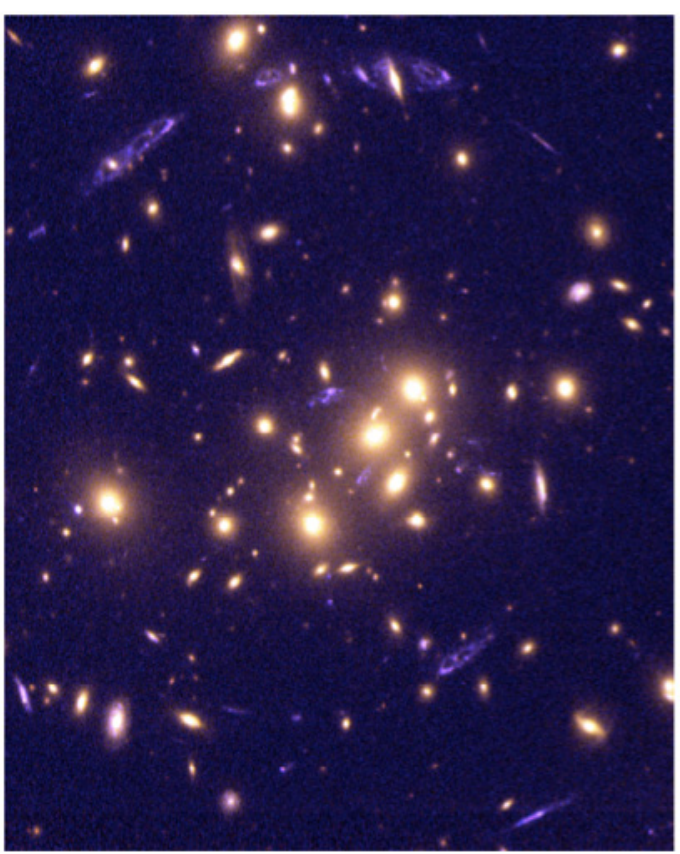

Figure 2

(Figure 1) A plot of stellar velocity vs. distance from galactic center, not shown is theoretically predicted velocity of objects as they move further from the galactic center. Figure obtained from [3].

(Figure 2) An image of weak gravitational lensing as described in [4]. In this Hubble image, the circular pattern of streaks can be seen, which is the telltale sign of gravitational lensing [5]. These blue shapes are really the same galaxy appearing from different points in an image, as described by the lensing phenomena. 
the image of the galaxy by either bending its shape or enlarging the image. In many cases, the object that the light is bent around is a cluster of galaxies, and the magnitude of the lensing effect can be used to determine the gravitational effect produced by the galaxy cluster. The image of the object that is being lensed may also appear in multiple places in an image as can be seen in Figure 2. In many observable cases of gravitational lensing, the lensing effect appears to be caused by objects with much greater mass than what the galaxy clusters appear to contain. This apparent discrepancy in observed mass and expected mass provides more evidence that inside these galactic superclusters, dark matter appears to contribute to the gravitational effect. The geometry behind the lensing effect is shown in Figure 3, where the light is being emitted from a source $S$, bent by the lens $L$, and observed by observer $O$. Because the path taken by the incoming light is unknown, it is observed to have originated from two separate sources $S_{1}$ and $S_{2}$. The amount of mass that is bending the incoming light can be calculated by determining the distance of the source and the angle at which the images appear to be separated by. Often when performing this technique, the amount of matter calculated to be causing the lensing effect is less than what is believed to be there from calculations of the apparent mass of the object. It is thought that this undetected mass is due to the presence of dark matter in the area of the lensing body. 


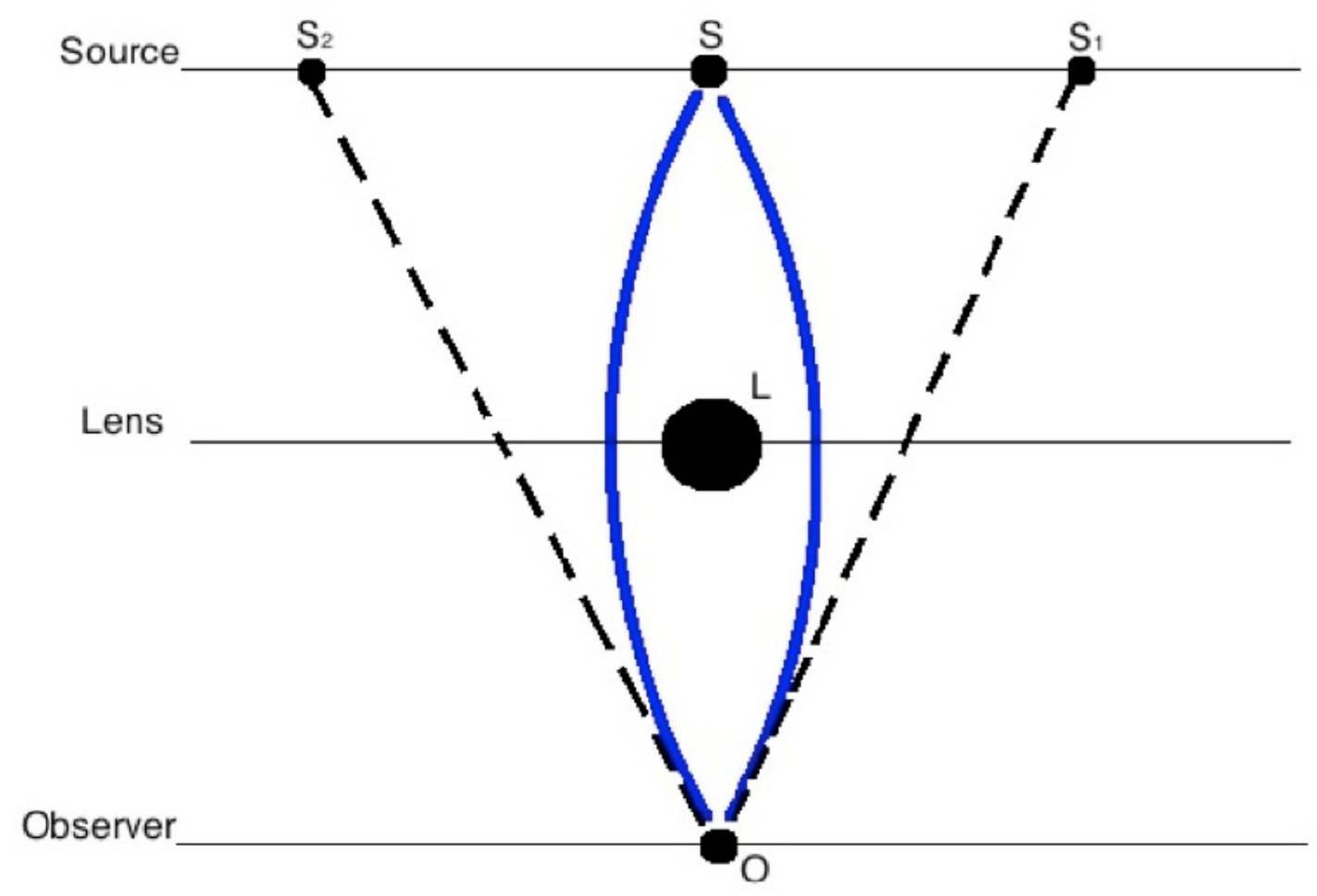

Figure 3

(Figure 3): A geometric image of how the lensing effect behaves. Incoming light from a source $S$ is bent around the lens $L$ towards the observer at $O$. This causes the source to appear to be in two different locations, at $S_{1}$ and $S_{2}$. Based on the angle between the two images and the distance from the observer, the mass of the lensing object can be determined.

Standard model particles interact with one another through four distinct forces: the gravitational force, the electromagnetic force, the weak force and the strong force [6]. The last three of these interactions are said to act through the exchange of force carrying particles; where photons carry the electromagnetic force, $\mathrm{W}$ and $\mathrm{Z}$ bosons carry the weak force that is responsible for the process of radioactive decay, and gluons carry the strong force between quarks within a nucleon and bind the nucleus together [6]. Current experiments are inconclusive as to the existence of a force-carrying particle for the gravitational interaction [6]. 
Much of nuclear and high-high energy physics experiments that are conducted today center around testing the validity and extending the breadth of the standard model. In this model there exist a number of various subatomic particles, each of which have their own properties of mass, spin, and charge. The particle collision experiments conducted at Jefferson Lab and labs all around the world involve attempting to either excite or break apart these tightly held quarks to determine properties of these particles and the forces that govern them.

\subsection{APEX}

The research conducted for this thesis is in relation to the A Prime Experiment in Hall A of Thomas Jefferson National Laboratory (JLab) and to assist with the end goals

of that experiment. Research was conducted in order to determine if equipment to be used would meet the required specifications that would allow for accurate and useful data collection. Research contributing to the thesis involved testing electronics that would be directly utilized with the detectors utilized in the right arm of the High Resolution Spectrometer (HRS).

\subsubsection{Theoretical Motivation}

The upcoming APEX experiment in Hall A at JLab seeks to uncover the existence of a new vector boson $A^{\prime}$, which is the hypothesized force carrier between dark matter particles, aptly named a "dark photon," and visible matter. It is also thought to interact through the weak force with standard model electrons. Through this interaction it should be possible to find evidence of Dark Matter. The APEX experiment will be conducted with the electron beam capabilities at Jefferson Lab. In the APEX experiment, $A$ ' dark photons may be produced in a process analogous to bremsstrahlung production. Electrons 
scattering off a target nucleus may give off an $A$ ' through electromagnetic interactions with the target nucleus [7]. The $A$ ' will subsequently decay into detectable standard model particles which will be detected in the spectrometers [7].

In the Standard Model, the only way for new forces to arise between particles is if charged particles were to acquire so called "millicharges," which allows the $A$ ' photon to undergo mixing with the Standard Model photon [7]. These forces would need to be sub$\mathrm{GeV}$ forces; and theoretical work and phenomenological expectations suggest that the $A$ ' dark photon will have a mass less than $1 \mathrm{GeV}$. For the APEX experiment, reactions in which the $A^{\prime}$ vector particle is produced by radiation off an electron beam, where the $A$ ' decays into an electron positron pair will be studied [7]. 


\section{Experimental Description}

\subsection{Electron Beam}

The accelerator facilities at JLab allow for the production of a highly polarized and luminous electron beam. At this time of the research conducted here, the accelerator site was capable of producing an electron beam with energy up to $6 \mathrm{GeV}$, at a current ranging from $10 \mathrm{nA}$ to $200 \mu \mathrm{A}$. Currently, construction is underway that will allow the facility to produce a beam with double the energy $(12 \mathrm{GeV})$ at the same rate as before. Currently there are three experimental halls (A, B, and C) which can run experiments independently. The current construction project, however, includes the addition of a fourth experimental hall (D).

Electrons are first introduced into the apparatus through the photoelectric effect, in which a $780 \mathrm{~nm}$ laser incident on a GaAs photocathode frees polarized electrons and accelerates them towards the first linear accelerator. The electrons are then accelerated by a series of linear accelerators and bent around the track with the use of magnets. Momentum is added by accelerating the electrons through the cryomodules in the linear accelerators with the use of radio waves of frequency $1497 \mathrm{MHz}$ [8]. When all three Halls are in operation, each hall receives beam every 2 nanoseconds (i.e. at a rate of 499 $\mathrm{MHz})$. 


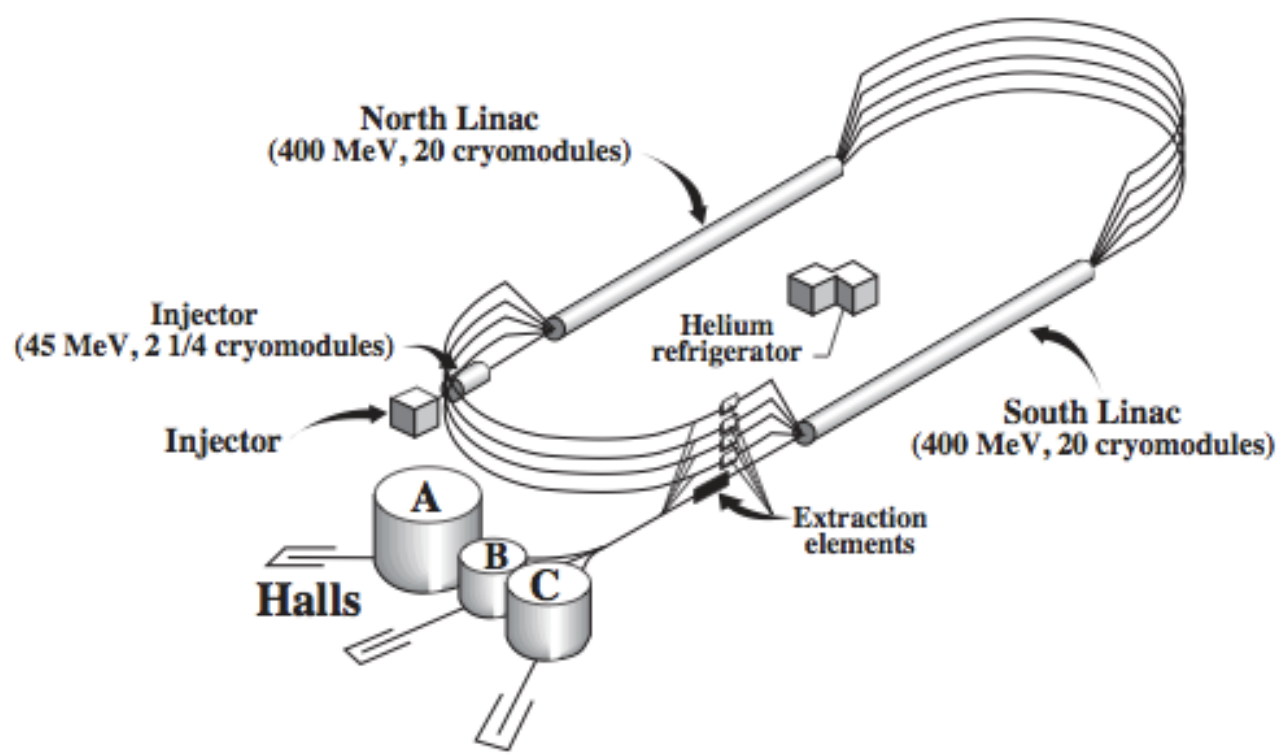

Figure 4: A schematic of Thomas Jefferson National Accelerator facility. Construction involves the addition of more cryomodules in the linear accelerator portions of the track and an additional hall at the end of the North Linac opposite to the Injector. Electrons pass five times through the track before exiting into one of the halls [9].

\subsection{Target}

The APEX experiment will measure the invariant mass spectrum of the electronpositron pairs that are created when the electron beam is scattered off the target. In this experiment, a target with high atomic number (i.e., heavy elements with a large nucleus and proton number) will be used [10]. Using four separate beam energies ranging from 1 to $4 \mathrm{GeV}$, the experiment will probe the $\mathrm{e}^{+} \mathrm{e}^{-}$spectra in the mass range of 65 to $550 \mathrm{MeV}$ $[10]$.

\subsection{Septum Magnet}

The focus of the APEX experiment will be on detecting scattered electron/positron pairs where each particle has approximately half the electron beam energy, and scatter off at very small angles compared to the incoming electron beam. 
While the geometry of the High Resolution Spectrometers (HRS) in Hall A of Jefferson Lab limits the smallest detectable scattering angle to be roughly 12 degrees, the addition of a septum magnet will allow the detection of scattered particles at four to five degree forward angles [10]. At these small angles, the scattered particles will have greater forward momenta, and their trajectory will be less influenced by the initial magnetic field the particles experience in the scattering chamber. The septum magnet is placed further down the beamline and serves to bend scattered particles further outward and into the spectrometers, more so than the initial magnet in the target chamber. These small angle particles have greater forward momenta, which causes them to undergo less deflection after scattering off the target. These particles have smaller scattering angles than would otherwise be obtainable by the detectors. Having a second magnetic field to alter the trajectory of the particles will allow for their detection as they are steered into the detector package. Without the septum magnet, the scattered particles of interest would not reach the detector package and instead travel forward towards the beam dump.

Figures 4 and 5 show a schematic of the septum magnet and a picture of the magnet during instillation.

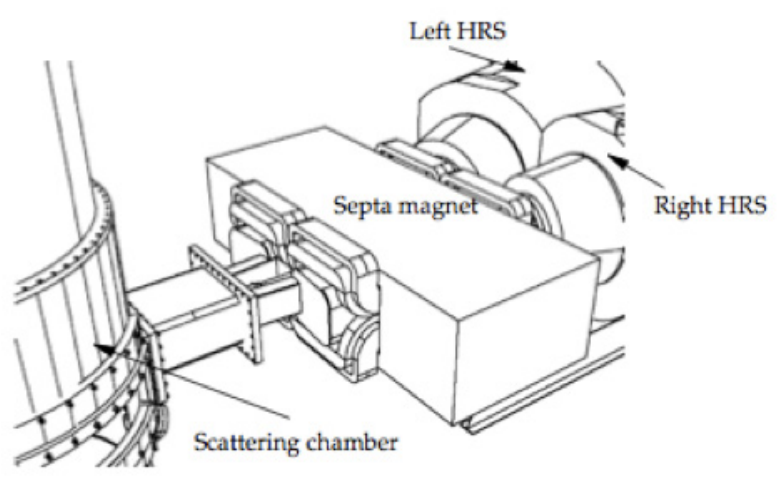

Figure 5

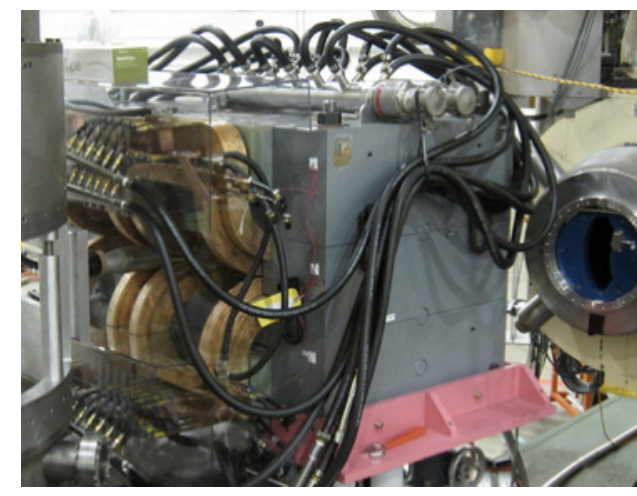

Figure 6

Figure 5: Shows a depiction septum magnet, beam enters from the left side and exits on the right into the two detector setups [7].

Figure 6: Shows a picture as the septum magnet was undergoing instillation in the hall for the PREX experiment [11]. 


\subsection{Detector Setup}

The detector package that will be utilized for APEX consists of two

spectrometers. The left and right arms are identical and consist of vertical drift chambers, scintillator counters, Aerogel Cherenkov detectors, a rear FPP chambers, a pion rejecter, and a two-layer shower calorimeter. For the research involved in this thesis, work was conducted with regards to only the vertical drift chambers and the shower calorimeters. Testing for A/D cards for the vertical drift chambers was conducted to assure proper functioning of equipment and expected output. Testing of summation modules was conducted for use in triggering for the shower counters. Figure 7 shows a general schematic of the detectors as they are installed in the hall, along with the location of DAQ electronics.

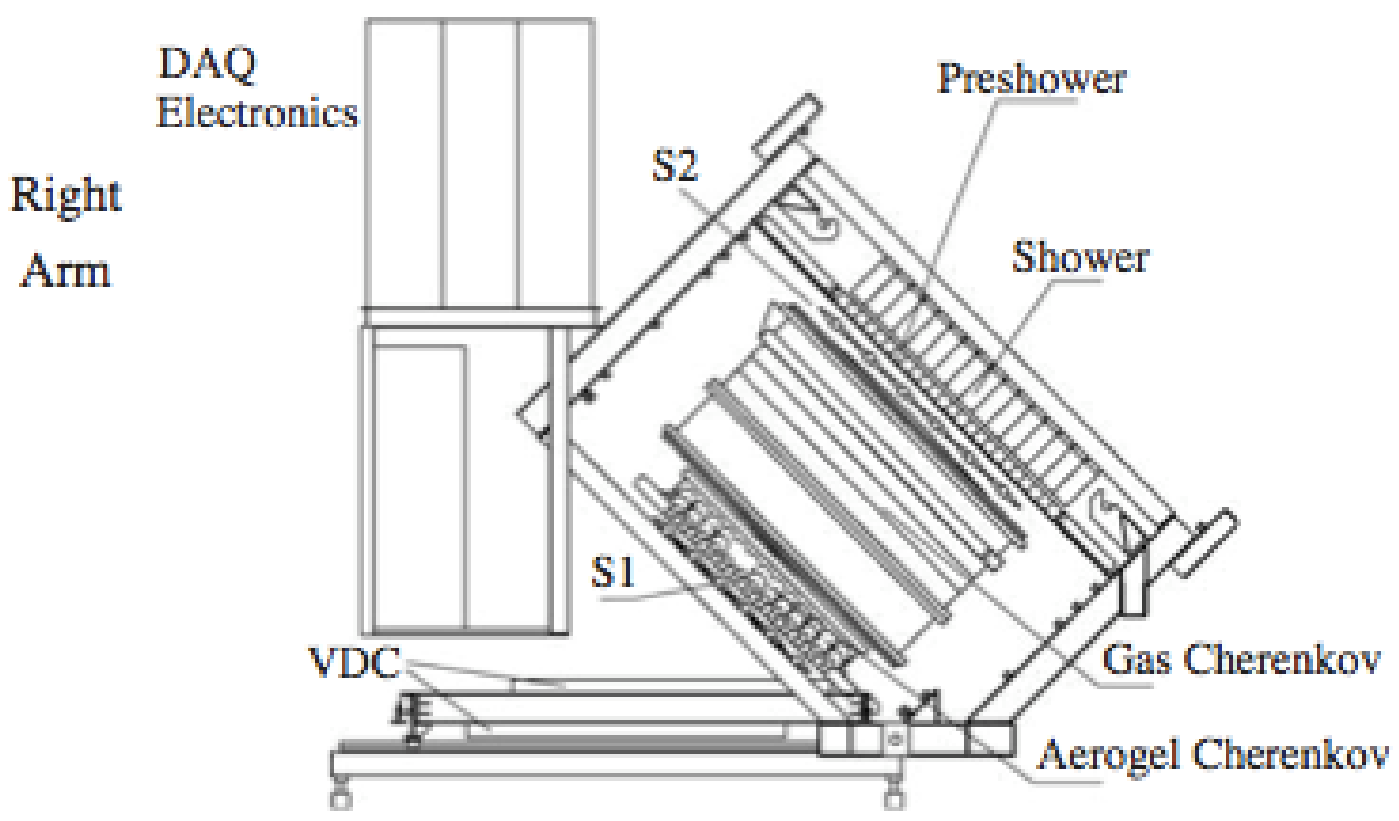

Figure 7: Depicted here is a schematic of the layout of detectors in the right arm of the HRS. Not shown is a similar arrangement for the left arm, which contains many of the same elements except for minor changes. For the concerns of this thesis, only the VDCs and the shower detectors are discussed any length. Picture courtesy of [9]. 


\subsubsection{Vertical Drift Chambers (VDCs)}

The particle tracking will be accomplished primarily through the use of the Vertical Drift Chambers (VDCs). Vertical Drift Chambers are commonplace for particle tracking purposes, and are one of the most effective methods for detecting particle position [9]. They provide very accurate detection to assist with the calculation of particle trajectories, with little contribution to loss of particle momentum or energy. Vertical Drift Chambers work by the use of many wires that are arranged adjacent to each other and are given a large negative potential. These wires are fixed in a chamber that is filled with a gas. When charged particle passes through the chamber it will ionize the gas within the chamber. When the gas is ionized the positive ion will move outward towards the edges of the chamber and the electron that was freed from the gas will be accelerated towards the wires. The electron will continue to collide with the gas and free more electrons as it accelerated towards the wire. This chain of collisions causes an amplification of signal, from one particle triggering an "avalanche" of particles that will collide with the wire and produce a detectable signal. The avalanche of particles allows for the detection of a single particle without needing to alter the energy of that particle drastically as it moves through the chamber. This process is the same general technique used by Geiger counters in detecting radiation. By using this technique with layers of wires with alternating orientations it becomes possible to determine the position of the particle as it moves through the detector. Each wire that gives a signal represents a position in space that the particle passed by as it moved through the detector. By using perpendicular layers of wires the three-dimensional trajectory can be reconstructed from the signals received. A 
picture of one of the VDCs before instillation is shown in Figure 8 without A/D cards installed.

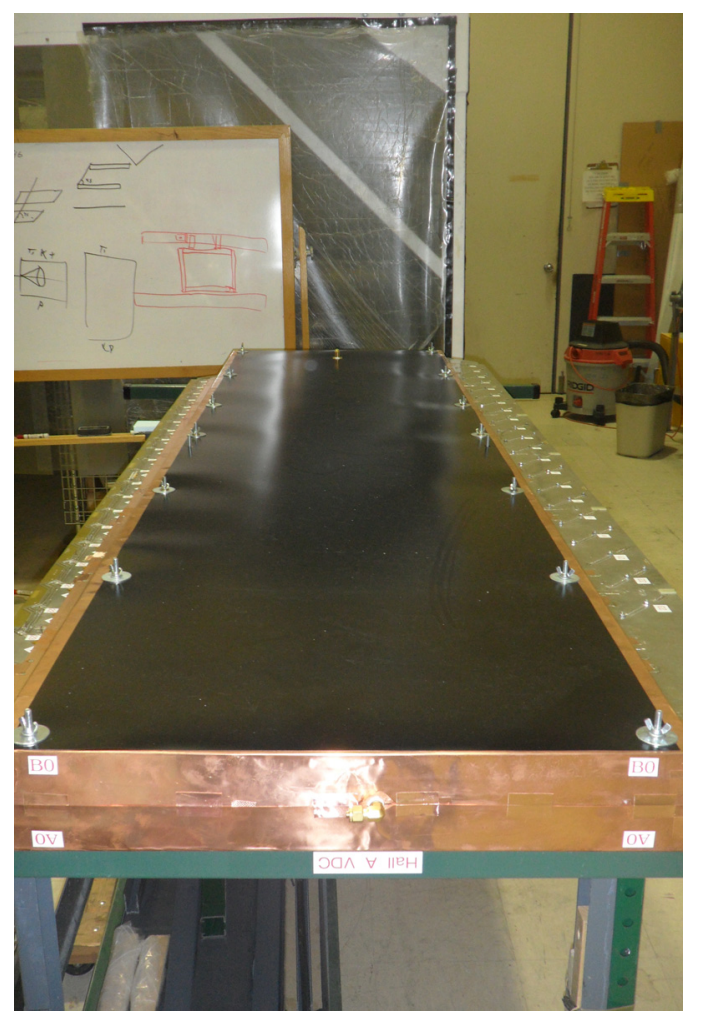

Figure 8: Picture of one of the Vertical Drift Chambers before installation in the hall and without A/D cards installed. A/D cards are installed along the edges of both of the longer portions of the drift chamber and receive up to 16 signals per card.

In the detector setup in use for the APEX experiment, VDCs are the first groups of detectors encountered after scattered particles leave the target chamber and pass through the septum magnet [8]. They are presents in both right and left arms of the HRS spectrometers in Hall A, and lie in a 45-degree angle with respect to the trajectory of the incoming particles as shown in Figure 9.

The VDCs each contain an array of wires oriented perpendicularly to each other and at 45 degrees with respect to the particle trajectory in both dispersive and nondispersive directions [12]. Utilizing two VDCs in tandem allows for very accurate 
trajectory mapping with the two planes separated by 0.335 meters. Measured drift velocities for each plane of wires was around $50.73 \pm 0.29$ micrometers per nanosecond and agreed with simulated values by less than $3 \%$ [12]. With a time resolution of 4.5 nanoseconds combined with the average drift velocity mentioned previously, the average position resolution for a given track is 225 micrometers. After determining two coordinates, the trajectory, and therefore the scattering angle, can be inferred. Figure 9 shows the rough path of particles as they travel through the VDCs, as well as the layout of wires in the chamber. 


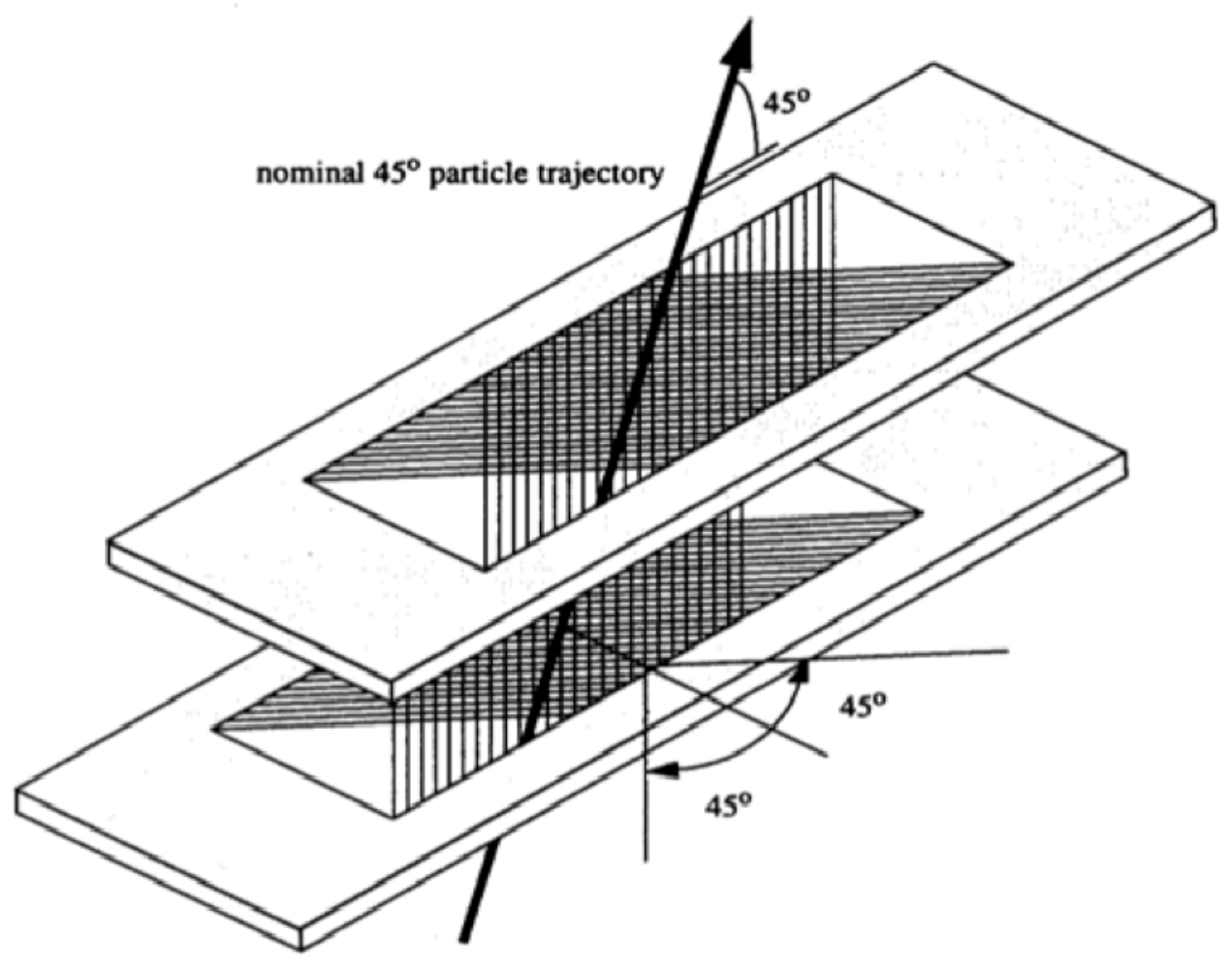

Figure 9: Depicts the two VDCs as they appear once installed. An imperfect alignment accounts for a horizontal distance displaced as scattered particles move towards the detector package. These detectors are parallel with the floor of the facility, while all other detectors are oriented as to have the frontal surface of the detector perpendicular with the incoming particles; the vertical drift chambers are the only detectors that are oriented parallel to the ornund Dirtura from $[121$

\subsubsection{Shower Counters}

The shower counters consist of two separate detector systems in tandem, an Electron Pre-Shower and a Total Absorber shower detector. Both of these detectors work using the same general principle. As an electron or positron transverses through a material, it will give off bremsstrahlung radiation. The particle will lose energy and the radiation emitted through this bremsstrahlung process will in turn ionize atoms in the material and subsequently free more electrons. These electrons will continue to emit 
bremsstrahlung radiation and free even more electrons. The emission of bremsstrahlung continues throughout the material until some minimum energy is reached where other effects dominate the bremsstrahlung method of energy loss. The process outlined here, however, serves not only to measure the energy of the particles that enter this section of the detector setup, but also serve to amplify the incoming signal from one electrons to millions before the signal reaches the data acquisition system.

The process of determining energy through bremsstrahlung emission is inherently different from the process described in the VDCs, where the particles create a cascade that is eventually detected by the wire. In this process, the energy of the traveling particle is in the end completely deposited in the detecting medium. In the VDC, the ionization of the gas is meant to minimally affect the particle momentum. In addition, the signal in the shower detectors is detected through the use of PMTs, where the cascading particles emit bremsstrahlung photons that reflect internally in the detector into the photocathode; but in the VDCs the signal is detected when ions collide with a wire and produce a current.

\section{Research Focus}

\subsection{Positron Trigger: Summing Electronics.}

The first task at hand was to setup a trigger that would allow the shower detectors to achieve an accurate energy resolution of scattered particles. The input signals into the summing modules would originate from Photomultiplier Tubes (PMTs) attached to 75 sections of the Total Absorber (TA) detectors and 48 Electron Pre Shower (EPS) detectors. By summing signals in groups of eight, and then summing the subsequent 
"summed" signals together, a total energy could be calculated from the summation of all signal received. Each module is equipped to handle up to 16 inputs, which produce two outputs of "summed" signals, which are then sent through four output channels located on the front of the module. These four outputs each send an identical signal comprised of the summed signals received from the eight input signals (Figure 6). Therefore, each module is capable of accepting 16 unique signals and producing from them 2 unique signals.

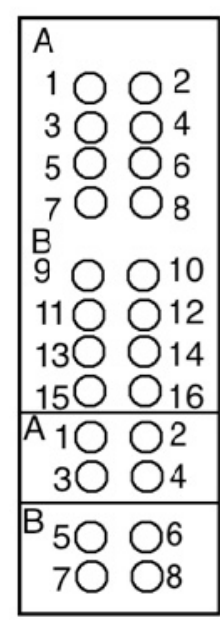

Figure 10

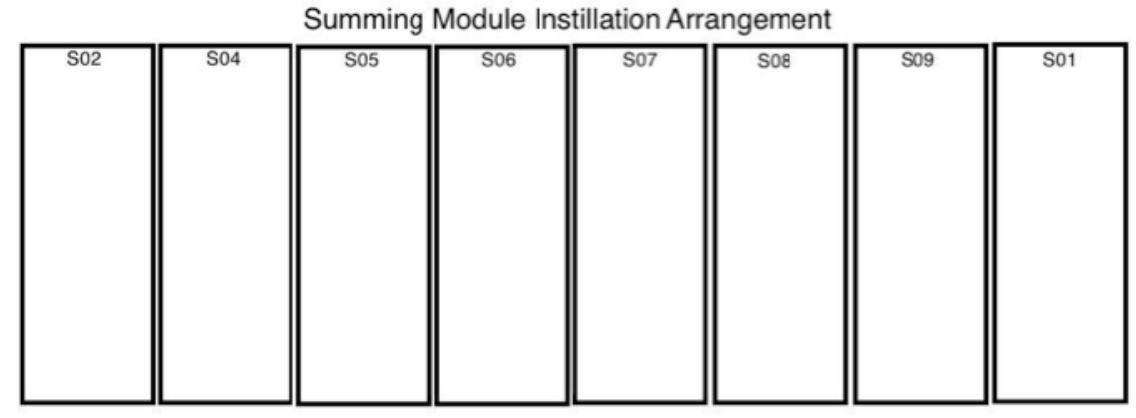

Figure 11

(Figure 10): Diagram of an individual summation module. The first two groups of eight inputs accept signals from EPS and TA detectors. These signals are output both as individual signals at the rear of the module and as summed signals in the front of the module. Group A on the bottom sums input signals one through eight, while group B sums signals nine through 16.

(Figure 11): Arrangement of the summation modules as they appear

The incoming signals were summed in groups of eight, i.e. EPSR 1-8, EPSR 9-16, EPSR 16-24, EPSL 1-8, etc; as well as TA 1-8, TA 9-15, etc. Channels TA 16, TA 32, TA 48, TA 64 , and TA 80 are empty channels that will receive no signals. Each module is capable of summing two groups, or 16 signals into two separate output signals. 
Channels one through eight of a given summing module add incoming signals linearly and provide an output in four output channels on the front of the module, on the modules these were usually labeled group A for both input and output channels. Similarly, channels nine through 16 were labeled as group B and the four output signals from these channels are labeled accordingly.

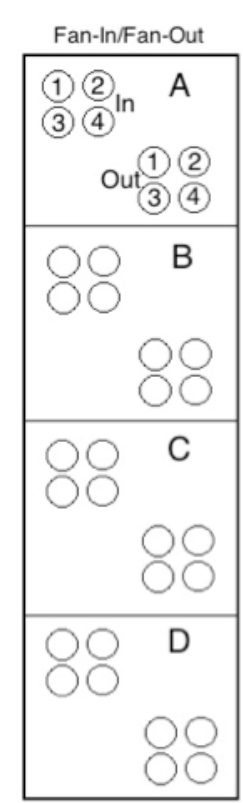

Figure 12

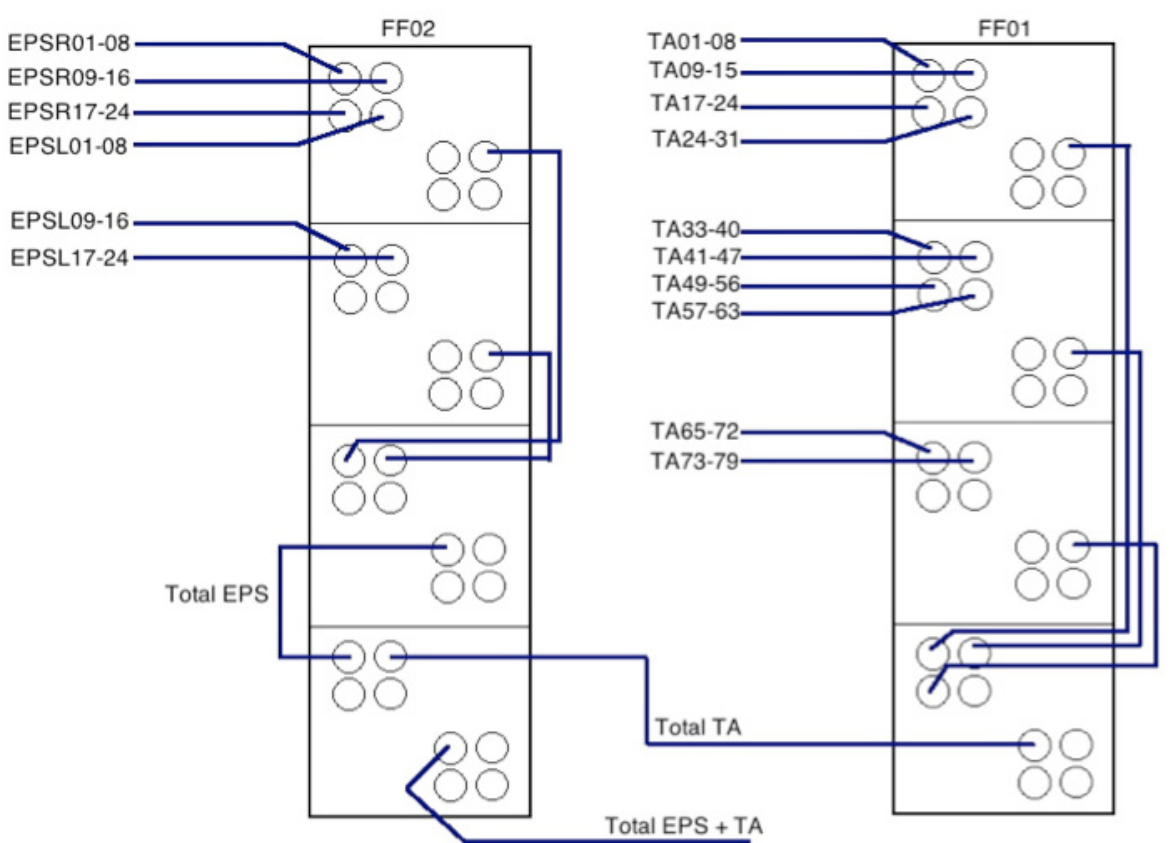

Figure 13

(Figure 12): Depiction of a single Fan-In/Fan-Out module, provides additional summation of signals and has four sections, each section taking four inputs and providing four identical outputs of the four input signals for that section.

(Figure 13): The summation arrangement for the signals. Signals originate in the summing modules and lead to the inputs of the two Fan-In/Fan-Out modules, total TA and total EPS are achieved separately and total signal from EPS and TA are achieved as well.

Signals sent from the summing modules were then routed to two Fan-In/Fan-Out modules for additional summation. These modules served to provide total summations for 
Electron Pre Shower and Total Absorber summations separately, as well as adding the two signals together and providing a total summation output of TA and EPS signals. The module is separated into four regions, each with four inputs and four outputs. Each of the four outputs provides a signal of the summation of the input signals for that section of the module. This arrangement is shown in Figures 8 and 9.

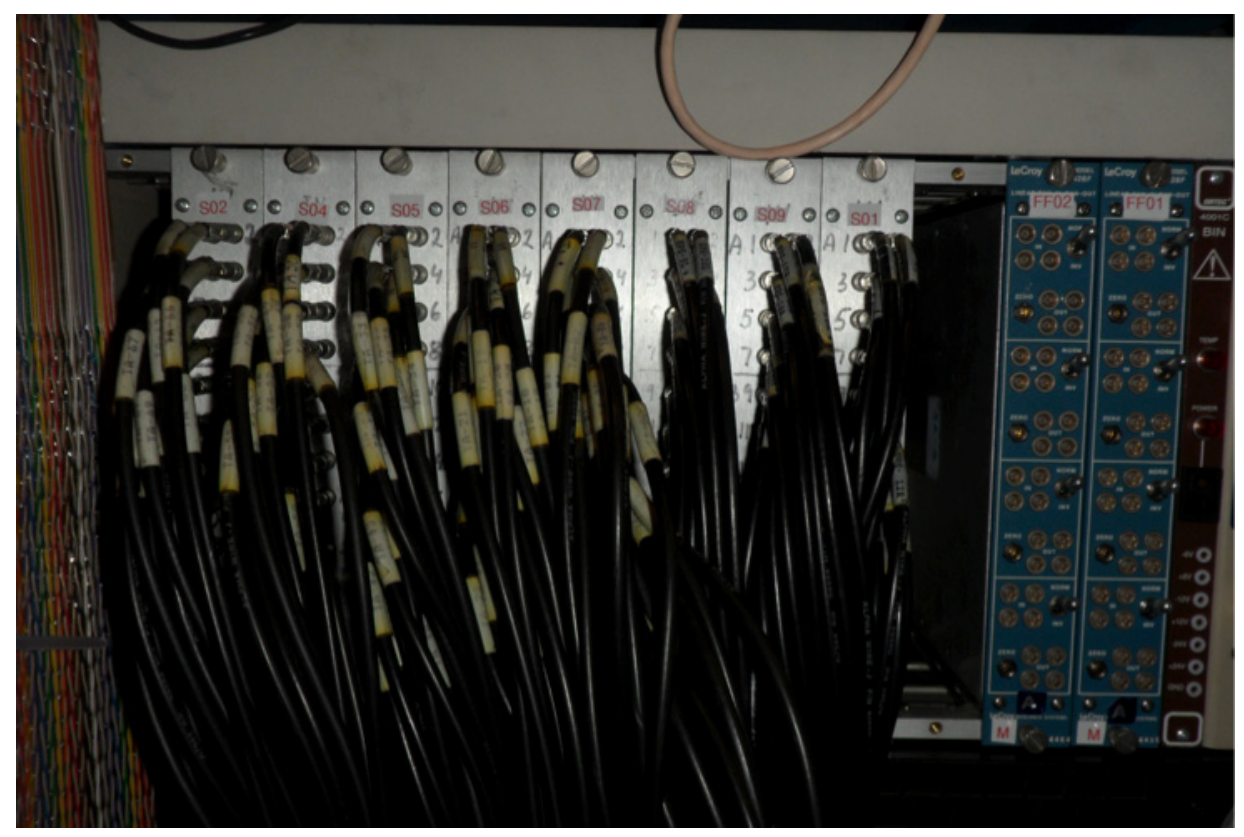

(Figure 14): Depiction of final arrangement of summing modules installed in the Hall, as depicted in Figure 7. The picture includes the cables from the TA and EPS inputs; the summing output arrangement is not depicted in this picture. The blue modules to the right are the Fan-in/Fan-out modules, used for summation of output signals from the summing modules themselves.

The final arrangement of input signals is shown in Figure 10. Incoming signals originate in EPS and TA shower detectors. Picture does not include cables connected into Fan-In/Fan-Out modules as depicted in Figure 9. Input signals connected to the FanIn/Fan-Out modules originate in the output channels of the modules shows, but are obscured by the wires. These outputs appear as described in Figure 6. Each module was 
tested for adequacy and proper functioning to assure optimal working condition for the experiment.

\subsection{Momentum Reconstruction: VDC Electronics.}

Because of the higher rates that are expected in the APEX experiment, new VDC amplification cards were needed to replace ones that are outdated and subsequently incapable of handling these increased rates. Initial tests show that compared to the old cards, the new cards are capable of operating at a threshold voltage five to ten times lower [13]. These cards were manufactured by the JLab Electronics Group and designed by the $\mathrm{G}^{\mathrm{n}}{ }_{\mathrm{E}}$ collaboration with the original intent of utilization in the multi wire drift chamber of the Big Bite Spectrometer.

The cards that have been selected for use in the APEX experiment were previously incorporated in the BigBite spectrometer used in Hall A. They have proven to be capable of handling the increased rates that the APEX experiment will produce. The cards replaced the previous versions, the LeCroy 2475DC and the Nanometric 277C, which were not capable of handling the high background rates that the BigBite spectrometer would be receiving [13].

With increased background signal rates, a significant leakage current in the chamber may be produced [13]. Having such a current would cause unstable operation of the drift chamber and a loss in the quality of the data obtained. By engineering a more stable A/D card with increased sensitivity to signal, the voltage can be decreased in the 
drift chamber. With a decrease in voltage, the background current induced should be decreased.

Increasing the sensitivity of the $\mathrm{A} / \mathrm{D}$ cards involves decreasing the minimum threshold voltage for the card. Thus, the new cards were engineered to have two features that would optimize the cards for the higher rates that are expected in the APEX experiment. First, the cards were outfitted with circuitry that reduced noise on the input circuit by switching from current-sensitive circuitry to charge-sensitive circuitry [13]. Second, the output signals are produced as low-voltage differential signaling (LVDS) signals, which produce less feedback than the otherwise used ECL signals that are standard on the LeCroy and Nanometric cards [13]. The new cards reduce required input voltage by a factor of five, reducing noise by a factor of five [7]. 


\section{Methods and Results}

\subsection{Equipment used.}

\subsubsection{Pulser}
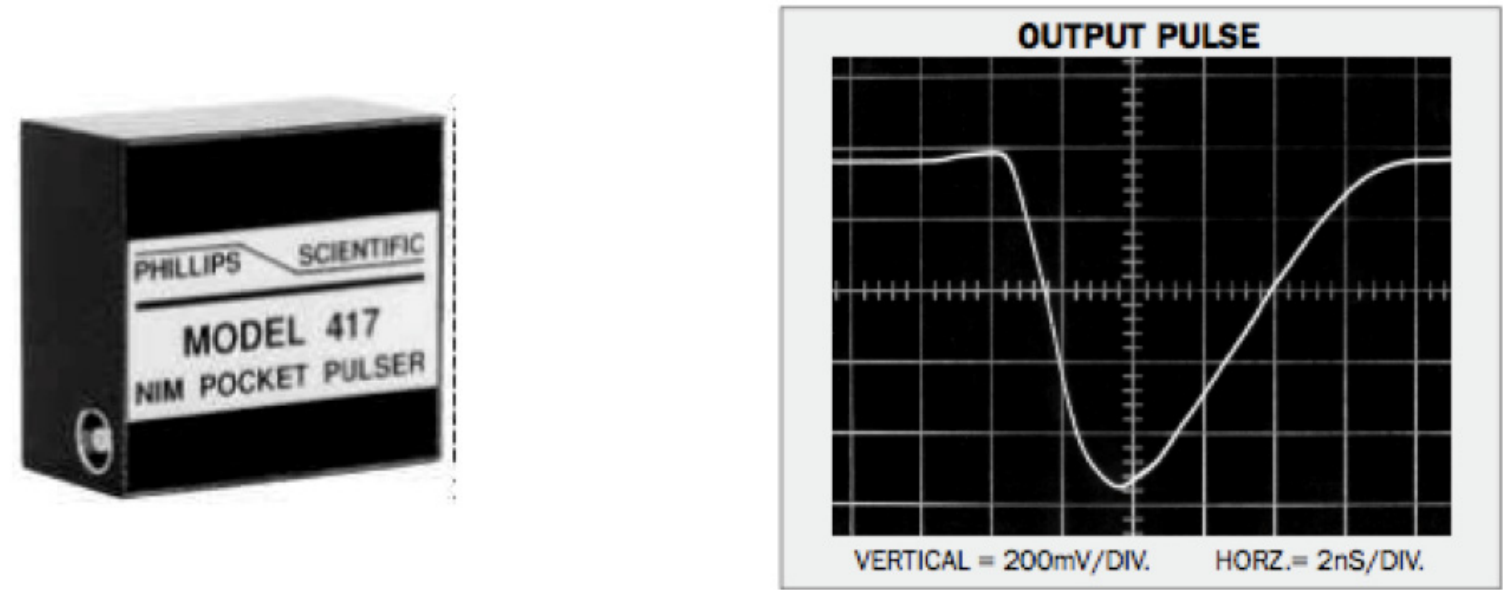

Figure 15

The Phillips Scientific Pocket Pulser on the left, produces a signal similar to that obtained from PMTs, this signal is depicted to the right. The scale on the graph shows around a $2 \mathrm{nS}$ rise time and a $6 \mathrm{nS}$ fall time [14].

To determine output voltages, a pulser was used to send a signal through each channel, and the output would be seen on the oscilloscope. The pulser is the Phillips Scientific NIM Pocket Pulser model number 417, which is used extensively throughout the research in the testing of both summation modules and of VDC A/D cards. It produces a signal specifically to mimic the signals received from PMTs in the negative 
range with amplitude of around $-800 \mathrm{mV}$ [14]. The signal has a 1.5 nanosecond rise time and about a 5 nanosecond fall time at a rate of about $10 \mathrm{KHz}$. Shown in Figure 13 is the image of the pulser and the shape of the signal produced. In practice the signal was heavily attenuated and discriminated to produce more manageable signals during testing.

\subsubsection{Discriminator}

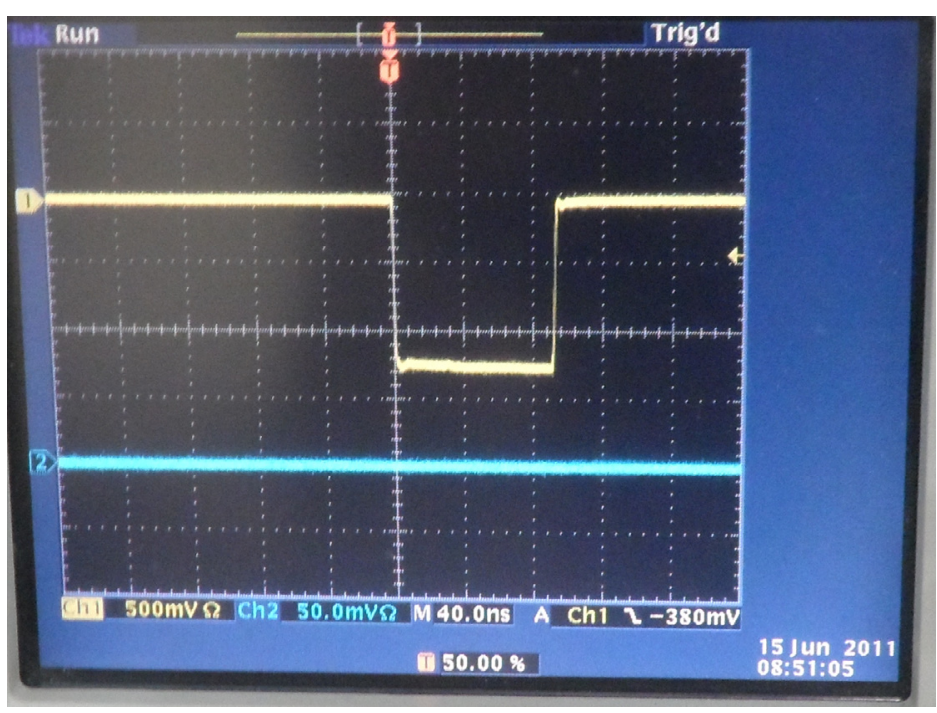

Figure 16

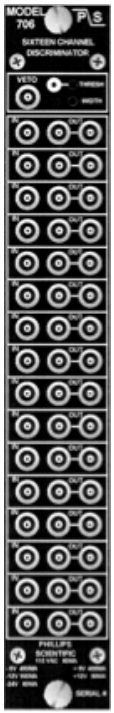

Figure 17

Figure 16: Signal received from discriminator during A/D card testing. A signal of about $-1200 \mathrm{mV}$ height and $100 \mathrm{~ns}$ width is produced.

Figure 17: Discriminator used in testing, contains 1 input and 2 outputs per channel. [15].

Discriminators are heavily used devices in High Energy and Nuclear physics experiments. They work by taking an analog signal and producing a digital signal only when the amplitude of the analog signal reaches a threshold [16]. The signal produced is of specific amplitude and signal width, which can be changed with the use of pulse shaping circuits. In testing of the VDC A/D cards, these signals were viewed on the oscilloscope to assure proper functioning of the cards, shown in Figure 16. 


\subsubsection{Attenuator}

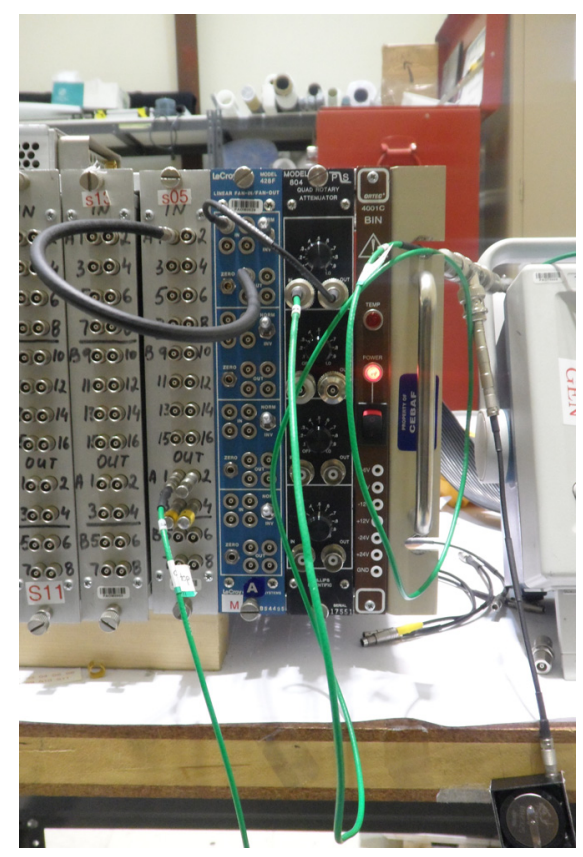

Figure 18

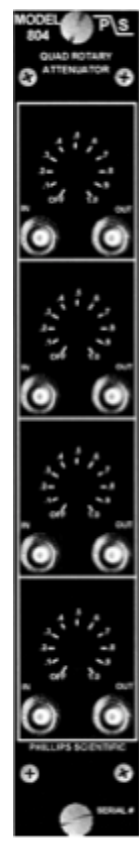

Figure 19

Figure 18: Setup for testing summation modules with the black attenuator located to the right of the blue module.

Figure 19: Closer view of the attenuator, showing the 4 identical and independent attenuation sections [17].

In some cases during data collection, it is necessary to alter the amplitude of an incoming signal. With a linear attenuator signal size is reduced uniformly. For studies outlined here, a Phillips Scientific NIM model 804 Quad Rotary Attenuator was utilized, which includes 4 separate sections of attenuation that operate independently. Attenuation 
occurs at 0.1 intervals from 0.1 to 1.0 attenuation. The attenuator also has the option of programming a custom level of attenuation or removing signal completely.

\subsubsection{Level Translator}

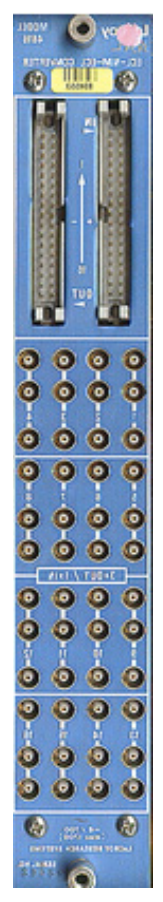

Figure 20: Image of the LaCroy NIM Model 4616 ECL/NIM/ECL Converter. In the studies described here, this device received signal from VDC A/D cards in the flat ribbon input at the top of the module and output through the LIMO cables to both scaler and oscilloscope [18].

A level translator was necessary in this setup in order to convert from NIM signals to emitter-coupled logic (ECL) signals. ECL has become popular in high-energy physics experiments, as it is the fastest form of digital logic available [16]. The level translator is necessary in order to convert the signal from the varying voltages between the NIM and ECL standards. While NIM signals can be either analog or digital, ECL 
signals are strictly digital. Digital signals have only two values, and act as a binary measurement to indicate if an event has or has not occurred; while analog signals can convey different information depending on various aspects of the signal such as size, shape, and speed. If a level translator is not used a misinterpretation of the data can result, where a positive event in one standard can be rejected in another or characteristics of the signal, such as shape and amplitude, may be destroyed.

\subsection{Testing of Summing Electronics}

\subsubsection{Schematic}

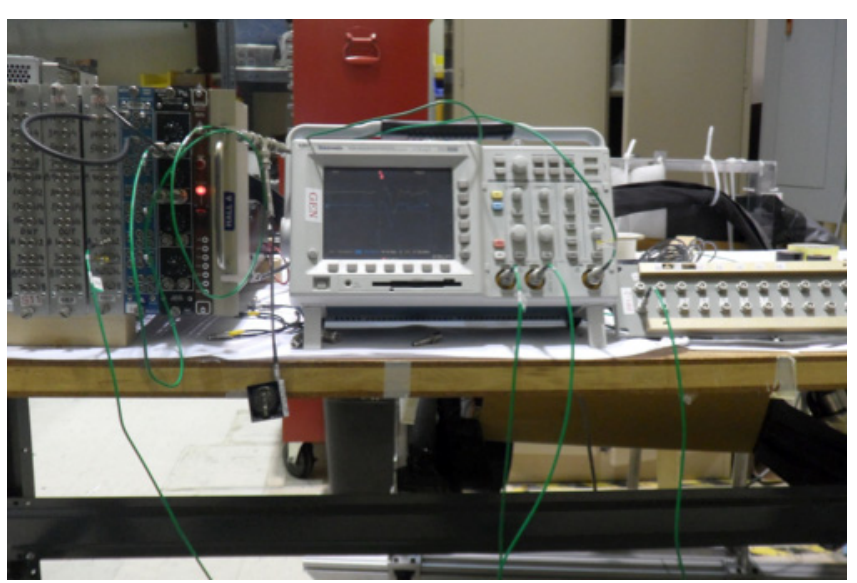

Figure 21

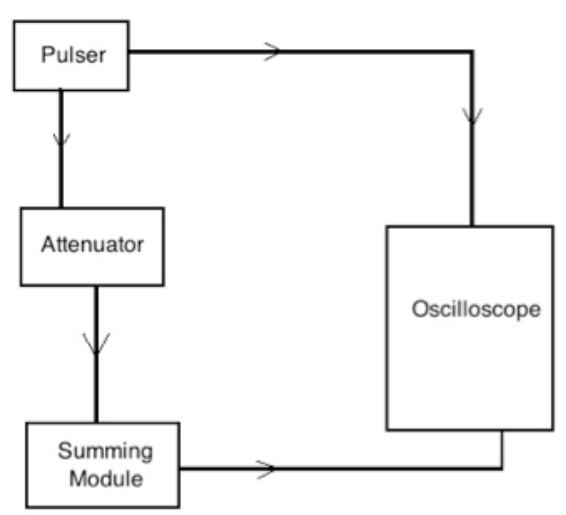

Figure 22

(Figure 21): The testing apparatus for determining output voltage and crosstalk for summing modules. Readings were taken from the oscilloscope by adjusting the trigger threshold voltage on the oscilloscope until the signal was visible on the screen; this was taken to be the output voltage for the channel.

(Figure 22): Schematic of the testing setup, signal from the pulser was split to both the summing module and the oscilloscope to provide a trigger to the

\subsubsection{Testing}

To construct the triggering process within the shower detectors, the summing modules used in the Hall needed to be tested to assure that they were in a working condition and that they would be capable of receiving the necessary signal and providing 
the correct output. The testing of summing modules consisted of two main goals. First, the output voltages of each channel needed to be tested to assure that the outbound pulse was of the expected size and shape. Second, an accurate description of the cross talk between adjacent channels needed to be documented to assure that the signal that was given to one channel was not showing up in significant levels in neighboring channels.

\subsubsection{Taking Measurements from the Summing Modules.}

Running the pulser signal through the input channel and feeding the output into the oscilloscope accomplished measurements of the exact output voltage on the channels. To obtain a measurement of the amplitude of the wave, the trigger level on the oscilloscope was adjusted until the signal from the pulser was just barely visible on screen. The trigger on the oscilloscope allows the oscilloscope to discriminate signals on its own, only picking up signals above a certain minimum voltage. The trigger would be adjusted until the signal from the pulser was no longer visible and then adjusted until the signal was just barely visible. At this point, it was determined that the oscilloscope was detecting signal from the pulser. Testing of the output voltages involved the pulser signal entering at the input on the front of the module and the oscilloscope connecting to each of the four summed output channels that would produce a signal from the corresponding input channel.

To determine cross talk, signal from the pulser would again be sent through an input channel on the front of the summing module. Unlike the summed output channels located at the front of the module, the single output channels located on the back of the module would produce signal that corresponded only to the signal for a specific channel. The single channel output is fed through a flat ribbon cable. Cross talk between channels 
was observed on the oscilloscope in a similar fashion to determining the output voltage.

In determining the crosstalk, an evaluation was made based on whether or not the value obtained reached some percent value of the threshold voltage. In general, if the total crosstalk on a single channel was less than ten percent of the threshold output voltage for that channel, the channel was deemed acceptable for the purposes of the experiment. In this regard, the amount of crosstalk was not determined explicitly with values recorded.

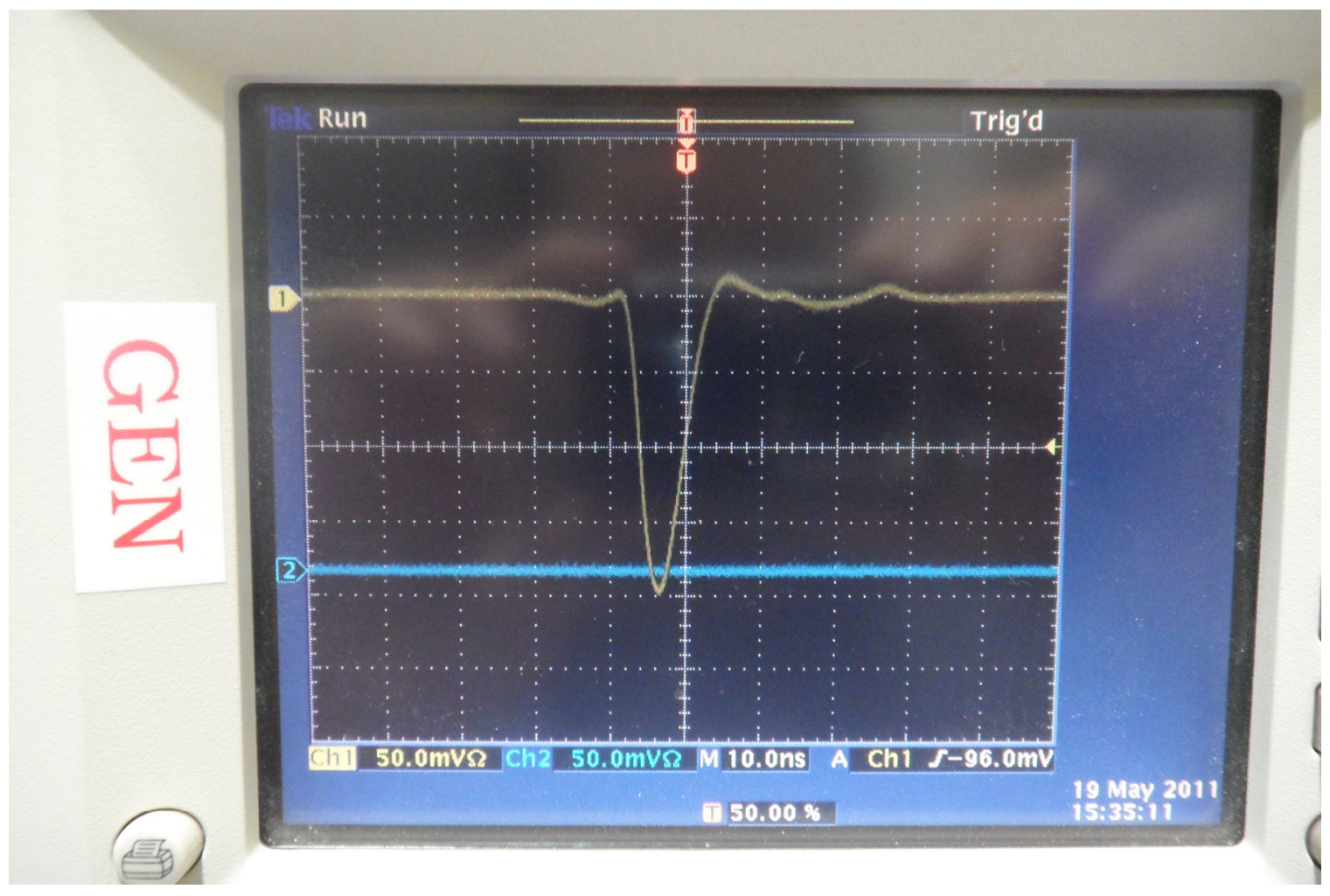

Figure 23: Picture of measured signal from summing modules. Incoming signal is produced by the Pulser and sent through the input on the front of the module. This signal appears from the summing output on the front, the blue line representing the trigger threshold is adjusted until the yellow line representing the signal just barely appears, if the blue line were adjusted to a point lower than shown no signal would be viewed on the oscilloscope.

\subsection{VDC Electronics}

Testing of VDC electronics consisted of collecting data on

Amplifier/Discriminator cards that will be utilized in the experiment. These cards were 
originally attached to the BigBite spectrometer, and due to their design characteristics, are useful for the APEX experiment.

\subsubsection{Signal Testing.}

Testing of VDC A/D amplification card signals was conducted in a similar manner to the testing of summing modules. In this test, determining threshold voltages for each channel and the crosstalk between channels was the primary focus. The setup for performing these tests was altered from the setup used to test the summing modules. The oscilloscope was used to determining the width of the analog signal that was received after running the pulse signal through the discriminator. To take voltage measurements, a DC voltage power supply provided a DC oltage to the A/D card, and readings were taken through the use of a scaler.

To test the VDC amplification cards, a pulser (as described in section 4.1.1) was used to create a uniform signal and was sent through a discriminator to an attenuator to lower the amplitude of the signal and to achieve the desired signal shaping. From the discriminator, signal was also routed to the scaler, which was used to make measurements and determine thresholds. From the attenuator signal was sent through the VDC amplification cards. The apparatus used to mount the A/D cards is capable of opening individual gates at the discretion of the user to send signal to individual channels of the VDC cards. From this apparatus, output leads from flat cable outputs of the VDC cards to the ECL-NIM level translator. Finally from this level translator the output is fed to another input of the scalar and to the oscilloscope. The apparatus that holds the VDC 
cards is powered by an independent DC power supply, which is imperative in determining threshold voltages.

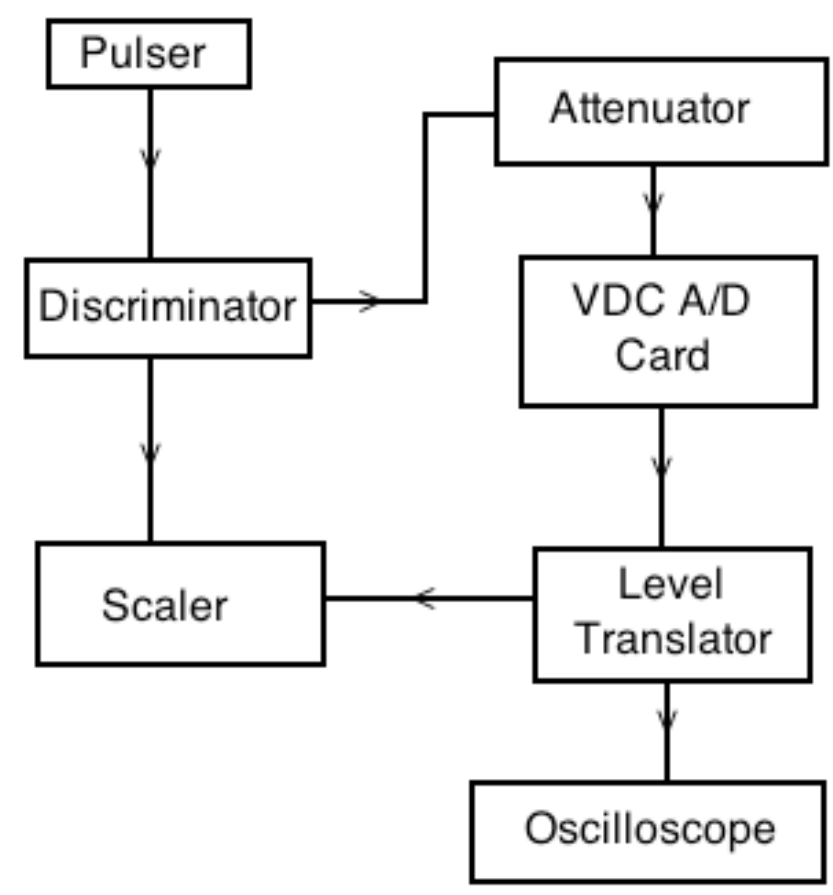

Figure 24: Diagram view of the path of signal originating from the pulser and moving throughout VCD card and analysis equipment. Connection from A/D card to level translator is through a rainbow ribbon cable. Not included in the figure is the DC power supply powering the VDC A/D card apparatus.

\subsubsection{NIM Standard}

Much of the equipment used in High Energy and Nuclear physics utilizes the NIM standard (Nuclear Instrument Module, mentioned earlier), which has been the earliest established system to provide a homogeneous style and structure to electronics equipment that is utilized in nuclear physics [16]. In this standard, the modules (discriminators, summation modules, amplifiers, etc.) are constructed to adhere to specific mechanical and electronic standards. Using this standard allows the equipment to 
be easily changed for use in other experiments and arranged in various ways to better adhere to the needs of the experimentalist [16]. In this standard, the modules are constructed to a height of $22.225 \mathrm{~cm}$, and a width of $3.43 \mathrm{~cm}$ [16]. Additionally, the module can appear in multiples of this width when necessary [16]. When the adequate set of modules has been determined, they can be installed into a so-called "NIM bin," which provides a standardized power voltage for the modules [16]. The NIM standard allows for the easily switching between modules, and equipment can be stored for later use or updated easily between other experiments utilizing the NIM standard. The NIM standard has since become a very widely adopted standard for use in nuclear and high-energy physics experiments worldwide [16].

To test the threshold voltages for each channel, the attenuator was set to $1 \%$ maximum signal by setting the first and second attenuator setting to $10 \%$. Signal from the attenuator would then be sent through the channel of the A/D cards one channel at a time. Then, the corresponding channel would be read from the level translator by the scalar. The scalar was adjusted to stop after reading 10k signals from the pulser and count signals received through the VDC card simultaneously. The voltage powering the VDC $\mathrm{A} / \mathrm{D}$ card apparatus was adjusted until the signal from the VDC card showed roughly $5 \mathrm{k}$ counts in the time it took the input directly from the discriminated pulser to read $10 \mathrm{k}$ counts. The voltage that produced $5 \mathrm{k}$ attenuated counts was recorded and deemed the threshold voltage.

To measure crosstalk, the $10 \mathrm{k}$ count limit on signals from the pulser was removed and the scalar was allowed to run with no limit, constantly taking counts from the pulser. Signal from the pulser would be allowed to run through a channel of the VDC 
amplification card and signal would be read from a neighboring channel. Signal would be sent through one channel of the VDC card, and be read through a neighboring channel from the level translator output to another channel of the scalar. The attenuation was adjusted until significant amplification was achieved, resulting in the neighboring channel detecting signal. The crosstalk value would be determined by making the attenuation adjustment as small as possible while still obtaining counts in neighboring channels. 


\section{Results and Discussion}

Due to the nature of the experiment, it was absolutely crucial that the APEX experiment utilized proper electronics that would be able to handle the increased rates expected during runtime. With the experiment utilizing such forward scattering angles, the rates that are expected in the APEX experiment are much greater than the rates at which previous electronics were able to handle. As stated earlier, the most effective way to assure that the equipment would be able to accept and interpret the increased flux of scattered particles was to lower the threshold voltage on the VDCs. By decreasing the gain on the wires in the VDC chamber the equipment becomes more sensitive to incoming signal and it is able to better handle the higher flux of background particles that are expected. In this sense, the equipment upgrade is pivotal in the search for evidence of dark matter.

The quantitative data and results of what has been outlined can be found in the attached appendices in tabular format along with instructions on how to interpret the results.

\subsection{VDC A/D Card results}

Vertical drift chamber amplifier/discriminator card testing proved to be quite cumbersome; however, the great majority of cards that were tested functioned well within allowed limits. Testing for minimum threshold voltage and input voltages were crucial in determining if the electronics were acceptable in the search for dark matter; as these values determined if the detectors would be capable of handling the expected increased 
rates. In the $\mathrm{A} / \mathrm{D}$ cards, crosstalk between channels was pivotal in assuring that accurate particle tracking could be accomplished. If signal through one wire induced a signal in a neighboring wire an inaccurate interpretation of particle trajectory would be recorded. Testing of the A/D cards was conducted as outlined in section 4.3.

Rejection criteria for the VDC A/D cards involved both inappropriate levels of crosstalk and unexpected levels of input and threshold voltage. Several cards experienced significant crosstalk, and were subsequently set aside to be repaired and to be implemented at a later time should the need arise. Most cards had threshold voltages between 2.3 and $2.8 \mathrm{mV}$, and crosstalk at 40 to 60 times threshold voltage. Additionally, if the signal shape appeared abnormal (i.e. the signal observed on the oscilloscope was not $100 \mathrm{~ns}$ in width, had an inappropriate amplitude, did not appear as a digital signal, or had inexplicable time-dependent behavior) the card was rejected to be repaired or discarded.

The results of the VDC A/D card threshold voltages are shown in Figure 25. The distribution appears to be a fairly normal distribution. There was an average threshold voltage of $2.57 \mathrm{~V}$ with a standard deviation of $0.07 \mathrm{~V}$. 


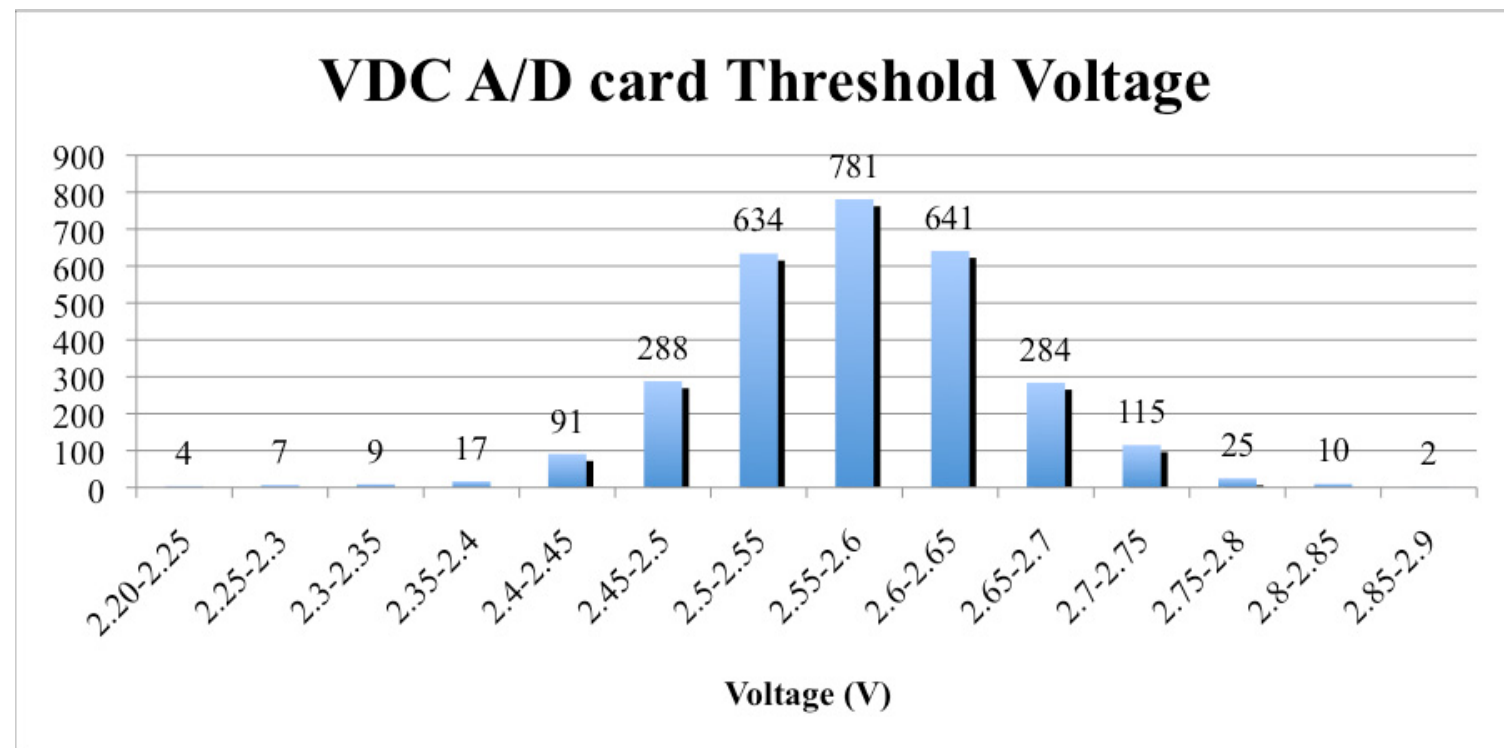

Figure 25: VDC A/D card threshold voltages presented as a frequency distribution.

Figure 26 shows a frequency distribution of the crosstalk data for the VDC A/D cards. The values of the horizontal axis represent the amount of amplification required to achieve crosstalk. A value of " 40 " mean that a signal 40 times larger than the threshold voltage is required to produce crosstalk in a channel. The average crosstalk was 47 times threshold voltage.

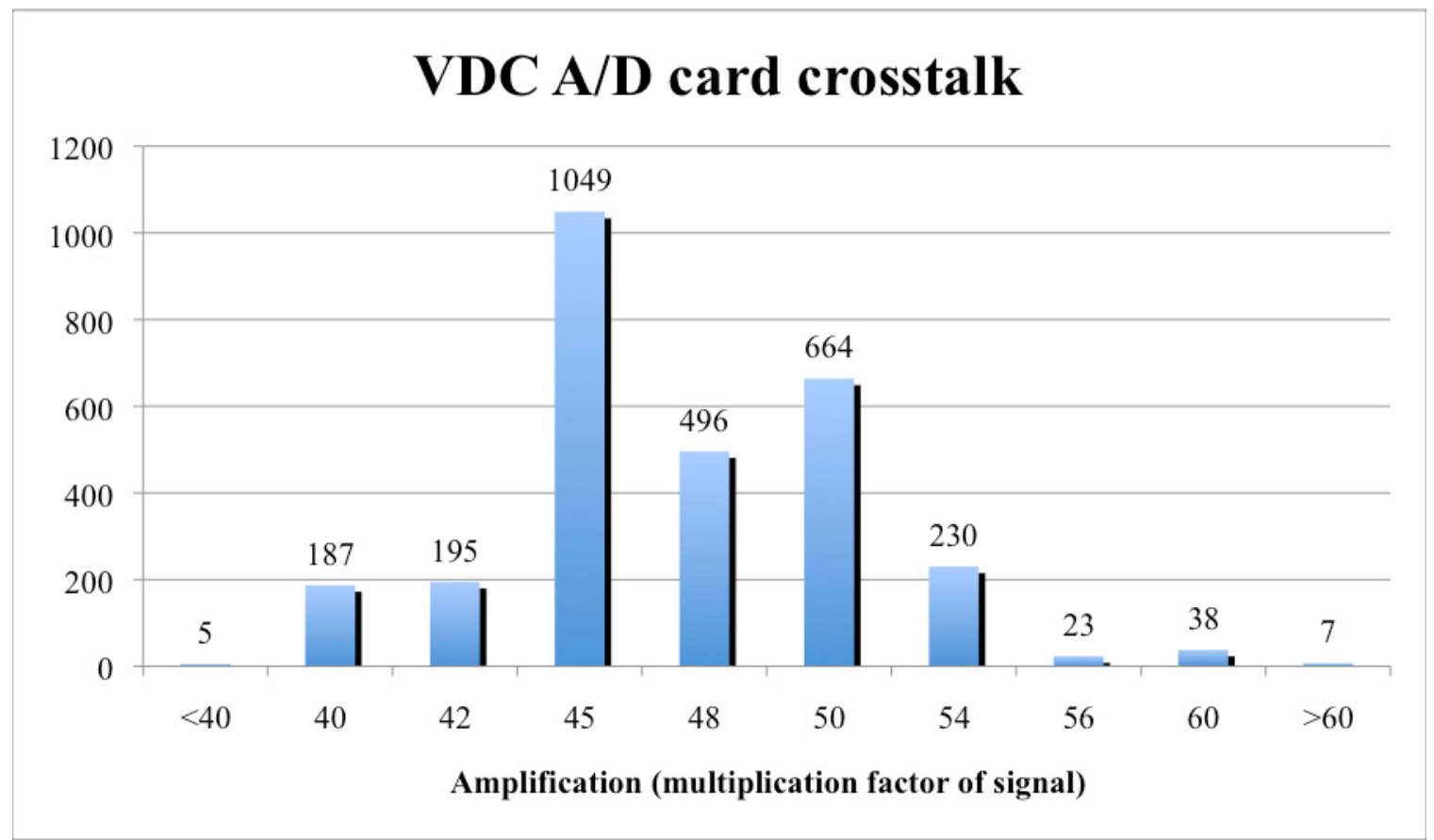


Figure 26: frequency distribution of crosstalk for VDC A/D cards.

\subsection{Summation Module results}

As with the testing of the VDC A/D cards, both crosstalk and threshold voltages were tested and measured for the summation modules. In the modules, crosstalk between channels would cause an inaccurate reading of particle momentum and energy. With an error in measuring particle energy as it is deposited in the shower counters, errors can occur in determining reaction kinematics and particle identification. Additionally, these errors could lead to inappropriate triggering of the data acquisition system, activating the storage of data that may otherwise not need to be collected and cause problems in data analysis later.

Testing involved the methods outlined in section 4.2, and focused on determining crosstalk relative to the incoming signal as well as comparing the input signal to the output signal for each channel to determine if there was significant signal loss. Large crosstalk, large signal loss, and strange time-dependent behavior in signal shape were used as criteria for rejection.

Initial data shows that most modules that were tested function as expected, with one module overheating, most likely due to a short circuit. All summing modules as well as fan-in/fan-out modules that were installed in the Hall operated well within the parameters that were outlined.

Testing of the modules produced several that operated within the expected threshold voltages and crosstalk limits. With an input voltage around $140 \mathrm{mV}$, channels produced output that ranged from 100 to $140 \mathrm{mV}$ from each channel. They were placed in the NIM crate and then installed in the hall near the detector package. They serve as some 
of the preliminary electronics that the signals from the detectors will encounter, and the signals from the TA and EPS shower detectors were connected to these summing electronics. Signals from these detectors were tested with the high voltage activated to determine if the detectors were still in working order. All high voltage systems appeared to be functioning without error.

The tests of cross talk showed that for most neighboring channels, the cross talk signal was less than two to three percent for each directly adjacent channel, and from one to two percent for channels located two locations away (i.e. reading channel 1, crosstalk from channel 2 was one location away, 3 was two locations away, ect.). This yields a total contribution from all neighboring channels of less than ten percent crosstalk per channel, a module that had more than ten percent crosstalk on any given channel was deemed unsatisfactory and set aside for repair.

Figure 27 shows a frequency distribution of the threshold voltages for the summation modules. The modules showed an average threshold voltage of $122 \mathrm{mV}$.

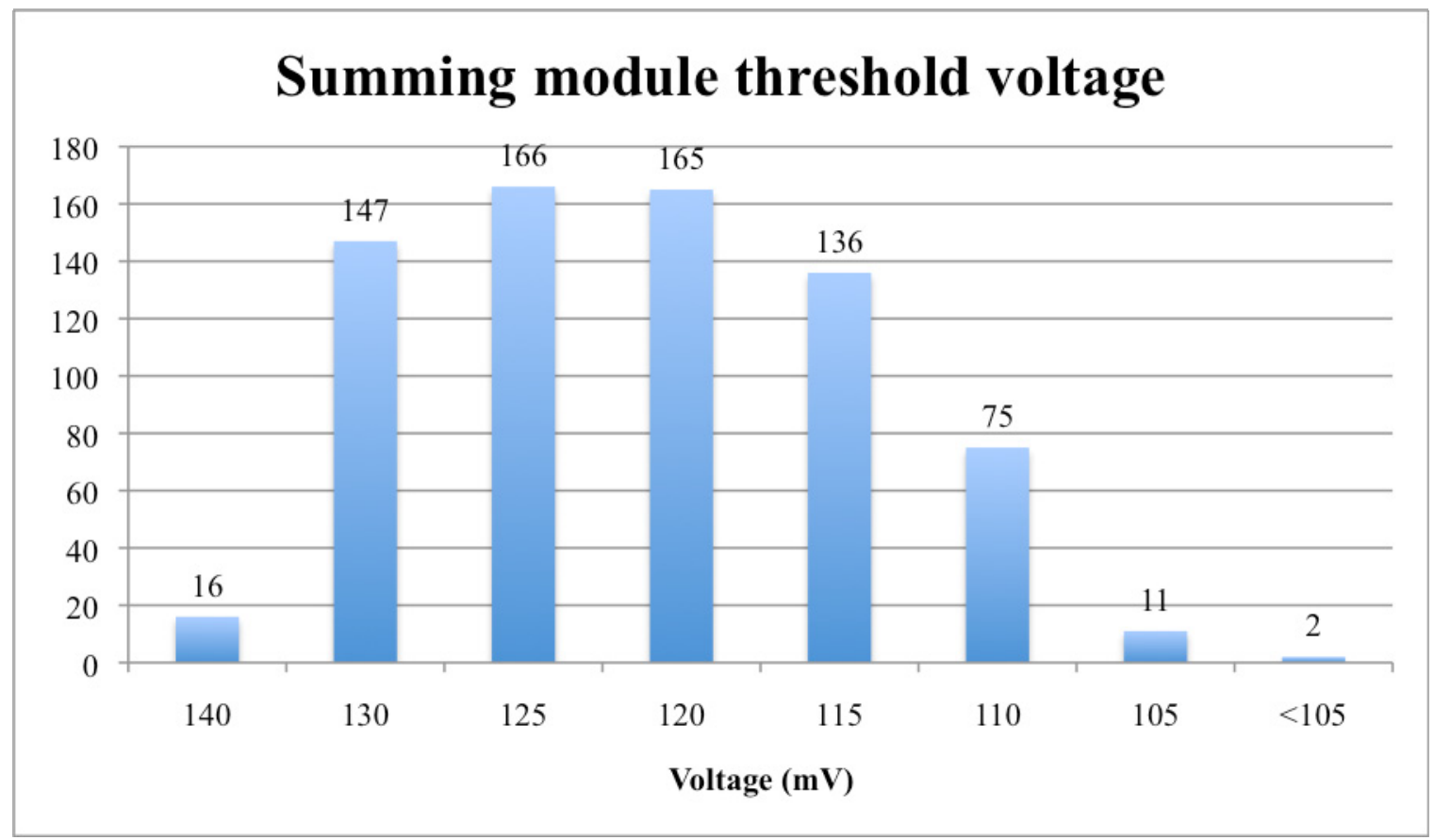


Figure 27: Frequency distribution for threshold voltages on channels in the summing modules.

\subsection{Conclusion}

With the analysis of the equipment discussed herein, it was determined that the electronics were still in proper working condition and still met the specifications necessary to allow for precise data collection and will allow the experiment to proceed as scheduled. With the VDC A/D cards operating as expected, the detector will be able to handle a higher rate of particles than was possible with previous equipment. With the summation modules tested and installed, an accurate trigger can be established to allow for particle identification. 


\section{References}

[1] G. Bertone, et al. (2005). Particle Dark Matter: Evidence, Candidates, and Constraints. Phys.Rept.405: 279-390.

[2] N. Arkani-Hamed, D. P. Finkbeiner, T. R. Slatyer, and N. Weiner, A Theory of Dark Matter, Phys. Rev. D79 (2009) 015014, [0810.0713].

[3] K.G. Begeman, HI rotation curves of spiral galaxies, Astronomy and Astrophysics, 223, 47-60, (1989).

[4] J. Wambsganss, (1998). Gravitational Lensing in Astronomy. Living Rev. Relativity. 1.12 .

[5] Space Telescope Science Institute, "Gravitational Lens Captures Image of Primeval Galaxy”, [Online HTML Document]: cited on 14 September 1998, http://oposite.stsci.edu/pubinfo/PR/96/10/A.html.

[6] R.A. Serway, C.J. Moses, C.A. Moyer. (2005). Modern Physics: third edition. Brooks Cole. pp. 548.

[7] R. Essig et al. (2010). The A' Experiment (APEX): Search for a New Vector Boson $A^{\prime}$ Decaying to $\mathrm{e}^{+} \mathrm{e}^{-}, \mathrm{A}$ Proposal to Jefferson Lab PAC37.

http://hallaweb.jlab.org/collab/PAC/PAC37/E12-10-009-APEX.pdf

[8] A.R. Acha, PhD thesis, Florida International University (2011).

[9] J. Alcorn et al. (2003). Basic Instrumentation for Hall A Nuclear Instruments \& Methods in Physics Research. Section A: Accelerators, Spectrometers, Detectors, and Associated Equipment, 522 (3). Pp. 294-346 
[10] R. Essig et al. (2010). An Electron Fixed Target Experiment to Search for a New Vector Boson $\mathrm{A}^{\prime}$ Decaying to $\mathrm{e}^{+} \mathrm{e}^{-}$.

[11] http://hallaweb.jlab.org/parity/prex/pics/prexMar10 023.jpg, http://hallaweb.jlab.org/parity/prex/, PREXII Experiment.

[12] K.G. Fissum et al. (2000). Vertical Drift Chambers for the Hall A High Resolution Spectrometers at Jefferson Lab. Jefferson Lab Technical Memorandum.

[13] I. Rachek, W. Gunning, C. Cuevas, and B. Wojtsekhowski et. al. (2007). Hall A Status Report - 2007.

[14] http://www.phillipsscientific.com/pdf/417ds.pdf

[15] http://www.phillipsscientific.com/pdf/706ds.pdf [16] W.R. Leo. (1994). Techniques for Nuclear and Particle Experiments: A How-to Approach. Springer-Verlag.

[17] http://www.phillipsscientific.com/pdf/804ds.pdf

[18] http://www.fnal.gov/projects/ckm/jlab/4616-spec.htm 


\section{Appendix A. Summation Module Testing}

These values are in units of milivolts. Columns with \# (\#) format are understood as inputs 1-8 correspond to outputs $1-4$; inputs $9-16$, outputs correspond to $5-8$, as is consistent with the summation module format.

\begin{tabular}{|c|c|c|c|c|c|c|c|c|c|c|}
\hline \multirow[t]{17}{*}{$\begin{array}{l}\text { Module } \\
\text { IN }\end{array}$} & & $\begin{array}{l}\text { S01 } \\
\text { OUT }\end{array}$ & & $1(5)$ & & $2(6)$ & & $3(7)$ & & \\
\hline & 1 & & 130 & & 130 & & 130 & & 130 & 130 \\
\hline & 2 & & 125 & & 125 & & 125 & & 130 & 130 \\
\hline & 3 & & 120 & & 120 & & 120 & & 120 & 120 \\
\hline & 4 & & 115 & & 110 & & 110 & & 115 & 115 \\
\hline & 5 & & 135 & & 125 & & 125 & & 130 & 130 \\
\hline & 6 & & 125 & & 125 & & 125 & & 125 & 125 \\
\hline & 7 & & 125 & & 120 & & 120 & & 120 & 120 \\
\hline & 8 & & 115 & & 115 & & 115 & & 115 & 115 \\
\hline & 9 & & 130 & & 140 & & 140 & & 130 & 135 \\
\hline & 10 & & 125 & & 130 & & 135 & & 125 & 130 \\
\hline & 11 & & 120 & & 125 & & 130 & & 120 & 120 \\
\hline & 12 & & 115 & & 120 & & 125 & & 110 & 115 \\
\hline & 13 & & 130 & & 135 & & 140 & & 125 & 130 \\
\hline & 14 & & 125 & & 130 & & 135 & & 125 & 125 \\
\hline & 15 & & 120 & & 120 & & 130 & & 115 & 120 \\
\hline & 16 & & 110 & & 115 & & 120 & & 110 & 115 \\
\hline \multirow{5}{*}{$\begin{array}{l}\text { Module } \\
\text { IN }\end{array}$} & & S02 & & & & & & & & \\
\hline & & OUT & & $1(5)$ & & $2(6)$ & & $3(7)$ & & \\
\hline & 1 & & 130 & & 125 & & 130 & & 135 & 135 \\
\hline & 2 & & 125 & & 120 & & 125 & & 135 & 130 \\
\hline & 3 & & 120 & & 115 & & 120 & & 125 & 125 \\
\hline
\end{tabular}




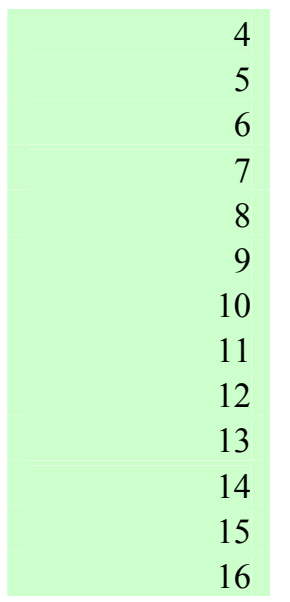

Module

S03

Overheated

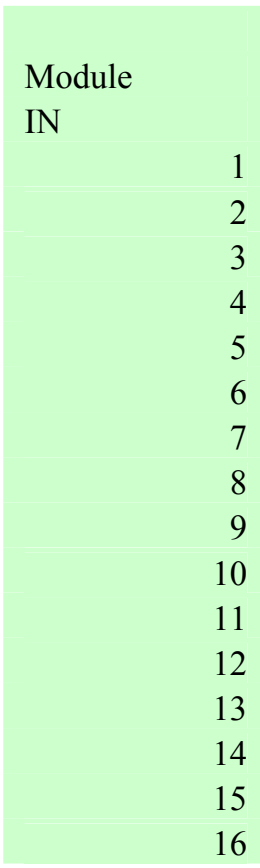

Module

IN

1

2

3

4

5

6

7

8

9

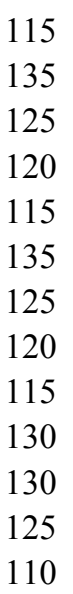

110

110
120
120
115
110
135
130
125
120
135
130
125
115

115

125

125

120

115

140

135

130

120

135

135

125

115
120

135

130

125

120

130

125

120

110

130

125

115

110
115

130

130

120

115

135

130

120

115

130

125

120

115
S04

OUT

130

130

120

114

135

125

125

115

135

130

120

115

130

130

125

110

S05

OUT

130

125

115

110

130

125

120

115

130
$1(5)$

2(6)
130
125

120

110

130

125

120

115

135

130

115

135

135

130

120

115

1 (5)

125

120

115

110

120

115

110

105

130
2 (6)

3(7)
130
130

125

115

130

125

120

115

140

135

120

135

135

130

125

120

2 (6)

125

120

115

110

120

120

115

110

135
$3(7)$

4(8)
140
135

130

125

140

135

125

120

125

120

110

125

125

120

115

105

3 (7)

135

130

120

115

130

125

120

115

125
135

135

125

120

135

130

120

120

130

125

110

130

130

125

120

110

4 (8)

130

125

120

115

125

125

120

115

130 


\begin{tabular}{|c|c|c|c|c|c|}
\hline 10 & 125 & 125 & 130 & 120 & 125 \\
\hline 11 & 115 & 115 & 125 & 110 & 115 \\
\hline 12 & 110 & 110 & 120 & 110 & 110 \\
\hline 13 & 130 & 130 & 135 & 120 & 125 \\
\hline 14 & 125 & 125 & 130 & 115 & 120 \\
\hline 15 & 120 & 115 & 125 & 110 & 115 \\
\hline 16 & 110 & 110 & 120 & 105 & 110 \\
\hline Module & S06 & & & & \\
\hline IN & OUT & $1(5)$ & $2(6)$ & $3(7)$ & $4(8)$ \\
\hline 1 & 130 & 125 & 130 & 135 & 135 \\
\hline 2 & 125 & 120 & 125 & 130 & 130 \\
\hline 3 & 120 & 115 & 115 & 125 & 125 \\
\hline 4 & 115 & 110 & 110 & 120 & 120 \\
\hline 5 & 130 & 120 & 125 & 130 & 130 \\
\hline 6 & 125 & 120 & 120 & 130 & 130 \\
\hline 7 & 120 & 110 & 115 & 120 & 120 \\
\hline 8 & 114 & 110 & 110 & 120 & 115 \\
\hline 9 & 135 & 135 & 140 & 130 & 130 \\
\hline 10 & 125 & 130 & 135 & 125 & 125 \\
\hline 11 & 115 & 120 & 125 & 115 & 115 \\
\hline 12 & 115 & 115 & 120 & 110 & 115 \\
\hline 13 & 130 & 130 & 140 & 125 & 130 \\
\hline 14 & 130 & 130 & 135 & 120 & 125 \\
\hline 15 & 125 & 120 & 125 & 115 & 120 \\
\hline 16 & 110 & 115 & 120 & 110 & 110 \\
\hline Module & S07 & & & & \\
\hline IN & OUT & $1(5)$ & $2(6)$ & $3(7)$ & $4(8)$ \\
\hline 1 & 130 & 125 & 130 & 135 & 130 \\
\hline 2 & 130 & 120 & 125 & 130 & 125 \\
\hline 3 & 120 & 115 & 115 & 125 & 120 \\
\hline 4 & 115 & 110 & 110 & 120 & 115 \\
\hline 5 & 135 & 125 & 125 & 135 & 130 \\
\hline 6 & 125 & 120 & 120 & 130 & 125 \\
\hline 7 & 125 & 115 & 120 & 125 & 120 \\
\hline 8 & 115 & 110 & 115 & 120 & 115 \\
\hline 9 & 135 & 135 & 140 & 125 & 130 \\
\hline 10 & 130 & 130 & 135 & 120 & 125 \\
\hline 11 & 120 & 120 & 130 & 115 & 120 \\
\hline 12 & 115 & 115 & 120 & 110 & 115 \\
\hline 13 & 130 & 130 & 135 & 125 & 130 \\
\hline 14 & 130 & 125 & 135 & 120 & 125 \\
\hline 15 & 120 & 115 & 120 & 110 & 115 \\
\hline 16 & 115 & 115 & 120 & 105 & 115 \\
\hline Module & S08 & & & & \\
\hline IN & OUT & $1(5)$ & $2(6)$ & $3(7)$ & $4(8)$ \\
\hline 1 & 130 & 130 & 135 & 140 & 135 \\
\hline
\end{tabular}




\begin{tabular}{|c|c|c|c|c|c|}
\hline 2 & 130 & 125 & 130 & 135 & 130 \\
\hline 3 & 120 & 120 & 125 & 130 & 125 \\
\hline 4 & 114 & 110 & 115 & 120 & 120 \\
\hline 5 & 135 & 125 & 130 & 135 & 135 \\
\hline 6 & 125 & 125 & 130 & 135 & 130 \\
\hline 7 & 120 & 115 & 120 & 125 & 125 \\
\hline 8 & 115 & 110 & 115 & 120 & 115 \\
\hline 9 & 135 & 135 & 140 & 130 & 135 \\
\hline 10 & 125 & 130 & 135 & 120 & 130 \\
\hline 11 & 120 & 125 & 130 & 115 & 120 \\
\hline 12 & 115 & 115 & 125 & 110 & 115 \\
\hline 13 & 130 & 135 & 140 & 130 & 135 \\
\hline 14 & 125 & 130 & 135 & 115 & 125 \\
\hline 15 & 120 & 120 & 125 & 115 & 120 \\
\hline 16 & 110 & 115 & 120 & 110 & 115 \\
\hline Module & S09 & & & & \\
\hline IN & OUT & $1(5)$ & $2(6)$ & $3(7)$ & $4(8)$ \\
\hline 1 & 130 & 125 & 130 & 135 & 130 \\
\hline 2 & 130 & 120 & 125 & 135 & 125 \\
\hline 3 & 120 & 115 & 120 & 130 & 120 \\
\hline 4 & 115 & 110 & 115 & 120 & 115 \\
\hline 5 & 135 & 125 & 130 & 135 & 130 \\
\hline 6 & 125 & 120 & 125 & 135 & 125 \\
\hline 7 & 125 & 115 & 120 & 125 & 120 \\
\hline 8 & 120 & 110 & 115 & 120 & 115 \\
\hline 9 & 135 & 135 & 140 & 125 & 130 \\
\hline 10 & 130 & 125 & 135 & 120 & 125 \\
\hline 11 & 120 & 120 & 125 & 115 & 115 \\
\hline 12 & 115 & 115 & 120 & 110 & 110 \\
\hline 13 & 130 & 130 & 135 & 125 & 125 \\
\hline 14 & 125 & 125 & 135 & 120 & 125 \\
\hline 15 & 125 & 120 & 125 & 115 & 115 \\
\hline 16 & 110 & 115 & 120 & 110 & 110 \\
\hline Module & $\mathrm{S} 10$ & & & & \\
\hline IN & OUT & $1(5)$ & $2(6)$ & $3(7)$ & $4(8)$ \\
\hline 1 & 110 & 100 & 100 & 110 & 105 \\
\hline 2 & 130 & 120 & 125 & 130 & 130 \\
\hline 3 & 120 & 115 & 120 & 130 & 125 \\
\hline 4 & 115 & 110 & 115 & 120 & 115 \\
\hline 5 & 130 & 120 & 125 & 135 & 130 \\
\hline 6 & 125 & 120 & 125 & 135 & 125 \\
\hline 7 & 120 & 115 & 115 & 125 & 120 \\
\hline 8 & 110 & 110 & 110 & 115 & 115 \\
\hline 9 & 130 & 135 & 140 & 125 & 130 \\
\hline 10 & 125 & 125 & 130 & 120 & 125 \\
\hline 11 & 125 & 120 & 125 & 115 & 120 \\
\hline 12 & 110 & 115 & 120 & 105 & 110 \\
\hline
\end{tabular}




\begin{tabular}{rrrrrr}
13 & 130 & 130 & 135 & 120 & 130 \\
14 & 125 & 125 & 130 & 120 & 125 \\
15 & 125 & 120 & 125 & 110 & 115 \\
16 & 110 & 115 & 120 & 105 & \\
\hline Module & & & & \\
IN & S11 & & & & \\
1 & OUT & $1(5)$ & $2(6)$ & $3(7)$ & 130 \\
2 & 130 & 120 & 125 & 135 & 125 \\
3 & 125 & 120 & 120 & 130 & 120 \\
4 & 120 & 110 & 115 & 125 & 115 \\
5 & 115 & 105 & 110 & 115 & 130 \\
6 & 130 & 120 & 125 & 130 & 125 \\
7 & 125 & 115 & 120 & 125 & 115 \\
8 & 120 & 110 & 115 & 120 & 130 \\
9 & 115 & 105 & 110 & 115 & 125 \\
10 & 130 & 135 & 140 & 125 & 115 \\
11 & 125 & 130 & 135 & 120 & 110 \\
12 & 120 & 115 & 125 & 115 & 125 \\
13 & 110 & 115 & 120 & 105 & 120 \\
14 & 130 & 130 & 135 & 120 & 115 \\
15 & 120 & 125 & 130 & 115 & 110 \\
16 & 120 & 120 & 125 & 110 &
\end{tabular}

\section{Appendix B. Fan-In/Fan-Out Module Testing}

Units are in milivolts, columns are the input channels and rows are the output channels. Readings were taken for each input/output combination.

\begin{tabular}{|c|c|c|c|c|c|c|c|c|}
\hline FF01 & in & & A & & & \multicolumn{3}{|c|}{ B } \\
\hline out & 1 & 2 & 3 & 4 & 1 & 2 & 3 & 4 \\
\hline 1 & 180 & 180 & 180 & 180 & 170 & 170 & 170 & 170 \\
\hline 2 & 180 & 180 & 180 & 180 & 175 & 175 & 175 & 175 \\
\hline 3 & 180 & 180 & 180 & 180 & 170 & 170 & 170 & 170 \\
\hline 4 & 180 & 180 & 180 & 180 & 175 & 175 & 175 & 175 \\
\hline & \multicolumn{4}{|c|}{ C } & & \multicolumn{3}{|c|}{$\mathrm{D}$} \\
\hline 1 & 180 & 180 & 180 & 180 & 165 & 165 & 165 & 165 \\
\hline 2 & 180 & 180 & 180 & 180 & 175 & 175 & 175 & 175 \\
\hline 3 & 180 & 180 & 180 & 180 & 165 & 165 & 165 & 165 \\
\hline 4 & 180 & 180 & 180 & 180 & 175 & 175 & 175 & 175 \\
\hline
\end{tabular}

\begin{tabular}{|c|c|c|c|c|c|c|c|c|}
\hline FF02 & in & & A & & & & B & \\
\hline out & 1 & 2 & 3 & 4 & 1 & 2 & 3 & 4 \\
\hline 1 & 165 & 165 & 165 & 165 & 160 & 155 & 155 & 155 \\
\hline 2 & 165 & 165 & 165 & 165 & 160 & 155 & 155 & 155 \\
\hline 3 & 165 & 165 & 165 & 165 & 160 & 155 & 155 & 155 \\
\hline 4 & 170 & 170 & 170 & 170 & 165 & 165 & 165 & 165 \\
\hline
\end{tabular}




\begin{tabular}{lllllllll} 
& \multicolumn{9}{c}{$\mathrm{C}$} & & \multicolumn{3}{c}{$\mathrm{D}$} \\
1 & 165 & 165 & 165 & 165 & 160 & 160 & 160 & 160 \\
2 & 165 & 165 & 165 & 165 & 160 & 160 & 160 & 160 \\
3 & 165 & 165 & 165 & 165 & 160 & 160 & 160 & 160 \\
4 & 170 & 170 & 170 & 170 & 165 & 165 & 165 & 165
\end{tabular}




\section{Appendix C. A/D Card testing results}

The following tables are organized by card number as they were labeled during testing. The columns indicate channel number on the card, with units of milivolts. The second table is an indication of the magnitude of amplification of incoming signal that is required to obtain crosstalk between neighboring channels. The final two columns of the second table are of the minimum internal threshold that is build into the card, which can be modified if needed, as well as an indication of whether the width of the pulse signal is as expected, a " 1 " being a positive indication.

Threshold

$\begin{array}{rrrrrrrrrrrrrrrrr} & \mathbf{1} & \mathbf{2} & \mathbf{3} & \mathbf{4} & \mathbf{5} & \mathbf{6} & \mathbf{7} & \mathbf{8} & \mathbf{9} & \mathbf{1 0} & \mathbf{1 1} & \mathbf{1 2} & \mathbf{1 3} & \mathbf{1 4} & \mathbf{1 5} & \mathbf{1 6} \\ 1 & 2.6 & 2.4 & 2.51 & 2.55 & 2.58 & 2.56 & 2.48 & 2.6 & 2.53 & 2.52 & 2.53 & 2.63 & 2.6 & 2.54 & 2.52 & 2.65 \\ 3 & 2.56 & 2.53 & 2.46 & 2.39 & 2.56 & 2.56 & 2.56 & 2.64 & 2.49 & 2.52 & 2.52 & 2.61 & 2.59 & 2.5 & 2.54 & 2.48 \\ 5 & 2.63 & 2.53 & 2.66 & 2.5 & 2.56 & 2.56 & 2.51 & 2.59 & 2.63 & 2.54 & 2.6 & 2.47 & 2.59 & 2.55 & 2.71 & 2.51 \\ 6 & 2.48 & 2.6 & 2.5 & 2.65 & 2.53 & 2.45 & 2.65 & 2.49 & 2.67 & 2.47 & 2.56 & 2.49 & 2.56 & 2.49 & 2.57 & 2.54 \\ 7 & 2.51 & 2.45 & 2.49 & 2.46 & 2.53 & 2.56 & 2.52 & 2.58 & 2.56 & 2.56 & 2.56 & 2.49 & 2.48 & 2.53 & 2.4 & 2.59 \\ 8 & 2.8 & 2.58 & 2.85 & 2.66 & 2.53 & 2.63 & 2.59 & 2.58 & 2.58 & 2.64 & 2.52 & 2.58 & 2.53 & 2.53 & 2.45 & 2.55 \\ 9 & 2.64 & 2.51 & 2.67 & 2.48 & 2.53 & 2.58 & 2.61 & 2.59 & 2.48 & 2.63 & 2.54 & 2.71 & 2.58 & 2.58 & 2.5 & 2.56 \\ 11 & 2.5 & 2.53 & 2.63 & 2.48 & 2.56 & 2.49 & 2.57 & 2.53 & 2.59 & 2.49 & 2.47 & 2.54 & 2.55 & 2.55 & 2.52 & 2.52 \\ 12 & 2.54 & 2.58 & 2.51 & 2.6 & 2.7 & 2.6 & 2.63 & 2.53 & 2.56 & 2.45 & 2.59 & 2.5 & 2.54 & 2.61 & 2.6 & 2.6 \\ 13 & 2.55 & 2.57 & 2.56 & 2.45 & 2.53 & 2.62 & 2.62 & 2.58 & 2.54 & 2.51 & 2.47 & 2.57 & 2.5 & 2.52 & 2.52 & 2.51 \\ 14 & 2.57 & 2.53 & 2.55 & 2.55 & 2.52 & 2.45 & 2.52 & 2.5 & 2.59 & 2.42 & 2.61 & 2.49 & 2.55 & 2.55 & 2.55 & 2.6 \\ 15 & 2.74 & 2.63 & 2.81 & 2.57 & 2.52 & 2.63 & 2.55 & 2.48 & 2.54 & 2.56 & 2.51 & 2.59 & 2.5 & 2.57 & 2.54 & 2.58 \\ 16 & 2.49 & 2.39 & 2.58 & 2.42 & 2.49 & 2.44 & 2.36 & 2.53 & 2.51 & 2.48 & 2.46 & 2.5 & 2.51 & 2.46 & 2.5 & 2.48 \\ 17 & 2.5 & 2.56 & 2.64 & 2.54 & 2.64 & 2.63 & 2.67 & 2.54 & 2.59 & 2.54 & 2.54 & 2.54 & 2.54 & 2.46 & 2.64 & 2.52 \\ 18 & 2.55 & 2.67 & 2.53 & 2.63 & 2.75 & 2.59 & 2.66 & 2.57 & 2.6 & 2.62 & 2.53 & 2.63 & 2.67 & 2.58 & 2.55 & 2.59 \\ 19 & 2.65 & 2.65 & 2.62 & 2.64 & 2.57 & 2.48 & 2.69 & 2.51 & 2.6 & 2.46 & 2.57 & 2.56 & 2.37 & 2.51 & 2.51 & 2.49 \\ 20 & 2.59 & 2.63 & 2.63 & 2.67 & 2.67 & 2.63 & 2.61 & 2.6 & 2.57 & 2.5 & 2.55 & 2.55 & 2.49 & 2.54 & 2.55 & 2.52\end{array}$




\begin{tabular}{|c|c|c|c|c|c|c|c|c|c|c|c|c|c|c|c|c|}
\hline 21 & 2.71 & 2.68 & 2.6 & 2.7 & 2.66 & 2.56 & 2.56 & 2.63 & 2.63 & 2.56 & 2.64 & 2.6 & 2.6 & 2.47 & 2.59 & 2.58 \\
\hline 22 & 2.69 & 2.58 & 2.73 & 2.58 & 2.68 & 2.66 & 2.56 & 2.6 & 2.58 & 2.72 & 2.59 & 2.69 & 2.57 & 2.42 & 2.6 & 2.53 \\
\hline 23 & 2.45 & 2.53 & 2.44 & 2.63 & 2.6 & 2.48 & 2.62 & 2.47 & 2.41 & 2.53 & 2.48 & 2.65 & 2.59 & 2.49 & 2.58 & 2.46 \\
\hline 24 & 2.6 & 2.49 & 2.56 & 2.56 & 2.49 & 2.47 & 2.64 & 2.52 & 2.4 & 2.53 & 2.59 & 2.47 & 2.37 & 2.43 & 2.47 & 2.47 \\
\hline 25 & 2.44 & 2.5 & 2.51 & 2.6 & 2.68 & 2.61 & 2.57 & 2.59 & 2.61 & 2.44 & 2.48 & 2.48 & 2.63 & 2.51 & 2.58 & 2.57 \\
\hline 26 & 2.52 & 2.58 & 2.58 & 2.6 & 2.54 & 2.49 & 2.63 & 2.67 & 2.56 & 2.58 & 2.48 & 2.69 & 2.47 & 2.51 & 2.51 & 2.6 \\
\hline 27 & 2.66 & 2.68 & 2.64 & 2.67 & 2.69 & 2.65 & 2.6 & 2.6 & 2.75 & 2.58 & 2.65 & 2.52 & 2.7 & 2.49 & 2.57 & 2.55 \\
\hline 28 & 2.62 & 2.64 & 2.41 & 2.57 & 2.52 & 2.6 & 2.61 & 2.63 & 2.6 & 2.73 & 2.47 & 2.63 & 2.65 & 2.68 & 2.65 & 2.52 \\
\hline 29 & 2.53 & 2.53 & 2.49 & 2.6 & 2.58 & 2.75 & 2.6 & 2.58 & 2.65 & 2.51 & 2.55 & 2.56 & 2.55 & 2.56 & 2.55 & 2.55 \\
\hline 30 & 2.56 & 2.64 & 2.57 & 2.57 & 2.51 & 2.52 & 2.48 & 2.59 & 2.64 & 2.5 & 2.5 & 2.51 & 2.58 & 2.56 & 2.54 & 2.54 \\
\hline 31 & 2.6 & 2.58 & 2.59 & 2.46 & 2.56 & 2.48 & 2.61 & 2.48 & 2.72 & 2.64 & 2.49 & 2.64 & 2.59 & 2.55 & 2.48 & 2.58 \\
\hline 32 & 2.64 & 2.5 & 2.58 & 2.58 & 2.65 & 2.45 & 2.55 & 2.51 & 2.55 & 2.53 & 2.46 & 2.7 & 2.66 & 2.49 & 2.64 & 2.51 \\
\hline 33 & 2.42 & 2.57 & 2.52 & 2.54 & 2.76 & 2.52 & 2.51 & 2.64 & 2.52 & 2.59 & 2.48 & 2.57 & 2.48 & 2.55 & 2.48 & 2.63 \\
\hline 34 & 2.67 & 2.53 & 2.54 & 2.55 & 2.79 & 2.51 & 2.63 & 2.51 & 2.54 & 2.59 & 2.5 & 2.55 & 2.65 & 2.47 & 2.7 & 2.6 \\
\hline 35 & 2.52 & 2.46 & 2.5 & 2.51 & 2.53 & 2.6 & 2.44 & 2.5 & 2.53 & 2.58 & 2.53 & 2.59 & 2.47 & 2.48 & 2.42 & 2.65 \\
\hline 36 & 2.54 & 2.59 & 2.67 & 2.53 & 2.55 & 2.64 & 2.7 & 2.62 & 2.72 & 2.5 & 2.67 & 2.53 & 2.62 & 2.65 & 2.53 & 2.53 \\
\hline 37 & 2.59 & 2.51 & 2.56 & 2.63 & 2.68 & 2.56 & 2.56 & 2.6 & 2.59 & 2.59 & 2.59 & 2.64 & 2.55 & 2.62 & 2.54 & 2.63 \\
\hline 38 & 2.71 & 2.52 & 2.53 & 2.56 & 2.6 & 2.6 & 2.66 & 2.64 & 2.76 & 2.6 & 2.67 & 2.56 & 2.62 & 2.51 & 2.54 & 2.56 \\
\hline 39 & 2.53 & 2.56 & 2.62 & 2.58 & 2.63 & 2.62 & 2.53 & 2.63 & 2.44 & 2.6 & 2.46 & 2.64 & 2.48 & 2.48 & 2.52 & 2.48 \\
\hline 40 & 2.65 & 2.6 & 2.68 & 2.67 & 2.63 & 2.66 & 2.61 & 2.66 & 2.62 & 2.65 & 2.57 & 2.52 & 2.55 & 2.55 & 2.5 & 2.58 \\
\hline 41 & $\begin{array}{l}2.64 \\
\text { BAD- } \\
\text { crossta }\end{array}$ & 2.57 & 2.49 & 2.56 & 2.49 & 2.55 & 2.51 & 2.62 & 2.48 & 2.45 & 2.48 & 2.58 & 2.57 & 2.59 & 2.52 & 2.48 \\
\hline 42 & lk & 2.56 & 2.59 & 2.66 & 2.57 & 2.63 & 2.63 & 2.72 & 2.62 & 2.53 & 2.59 & 2.69 & 2.55 & 2.59 & 2.56 & 2.45 \\
\hline 43 & 2.52 & 2.63 & 2.57 & 2.57 & 2.55 & 2.6 & 2.59 & 2.62 & 2.63 & 2.5 & 2.57 & 2.57 & 2.47 & 2.5 & 2.51 & 2.48 \\
\hline 44 & 2.64 & 2.62 & 2.6 & 2.69 & 2.65 & 2.75 & 2.7 & 2.74 & 2.71 & 2.57 & 2.82 & 2.64 & 2.61 & 2.59 & 2.6 & 2.54 \\
\hline 45 & 2.69 & 2.58 & 2.5 & 2.65 & 2.62 & 2.54 & 2.54 & 2.63 & 2.73 & 2.61 & 2.7 & 2.6 & 2.55 & 2.53 & 2.53 & 2.55 \\
\hline 46 & 2.62 & 2.67 & 2.7 & 2.68 & 2.64 & 2.7 & 2.58 & 2.61 & 2.6 & 2.63 & 2.62 & 2.62 & 2.6 & 2.54 & 2.65 & 2.58 \\
\hline 47 & 2.51 & 2.75 & 2.46 & 2.67 & 2.67 & 2.55 & 2.63 & 2.58 & 2.58 & 2.5 & 2.59 & 2.51 & 2.53 & 2.53 & 2.47 & 2.55 \\
\hline 48 & 2.58 & 2.68 & 2.64 & 2.6 & 2.6 & 2.6 & 2.63 & 2.55 & 2.68 & 2.55 & 2.62 & 2.55 & 2.6 & 2.58 & 2.53 & 2.47 \\
\hline 49 & 2.56 & 2.62 & 2.59 & 2.55 & 2.61 & 2.61 & 2.61 & 2.47 & 2.58 & 2.49 & 2.45 & 2.52 & 2.63 & 2.56 & 2.67 & 2.6 \\
\hline 50 & 2.64 & 2.65 & 2.59 & 2.59 & 2.61 & 2.49 & 2.64 & 2.55 & 2.55 & 2.64 & 2.48 & 2.61 & 2.65 & 2.49 & 2.62 & 2.53 \\
\hline 51 & 2.4 & 2.61 & 2.45 & 2.64 & 2.55 & 2.53 & 2.65 & 2.59 & 2.68 & 2.6 & 2.72 & 2.67 & 2.54 & 2.65 & 2.64 & 2.62 \\
\hline
\end{tabular}




\begin{tabular}{|c|c|c|c|c|c|c|c|c|c|c|c|c|c|c|c|c|}
\hline 52 & 2.7 & 2.48 & 2.67 & 2.6 & 2.62 & 2.67 & 2.85 & 2.68 & 2.66 & 2.66 & 2.55 & 2.62 & 2.61 & 2.56 & 2.64 & 2.47 \\
\hline 53 & 2.62 & 2.49 & 2.63 & 2.49 & 2.6 & 2.6 & 2.51 & 2.6 & 2.48 & 2.5 & 2.42 & 2.58 & 2.57 & 2.63 & 2.57 & 2.62 \\
\hline 54 & 2.44 & 2.63 & 2.56 & 2.62 & 2.65 & 2.54 & 2.65 & 2.57 & 2.53 & 2.53 & 2.53 & 2.57 & 2.63 & 2.62 & 2.51 & 2.48 \\
\hline 55 & 2.65 & 2.61 & 2.61 & 2.68 & 2.68 & 2.51 & 2.68 & 2.54 & 2.59 & 2.53 & 2.63 & 2.57 & 2.52 & 2.52 & 2.51 & 2.66 \\
\hline 56 & 2.57 & 2.45 & 2.58 & 2.53 & 2.56 & 2.49 & 2.51 & 2.47 & 2.55 & 2.55 & 2.55 & 2.54 & 2.51 & 2.6 & 2.55 & 2.69 \\
\hline 57 & 2.65 & 2.5 & 2.6 & 2.62 & 2.55 & 2.57 & 2.55 & 2.6 & 2.57 & 2.54 & 2.56 & 2.64 & 2.52 & 2.5 & 2.5 & 2.57 \\
\hline 58 & 2.58 & 2.55 & 2.57 & 2.57 & 2.61 & 2.58 & 2.51 & 2.48 & 2.67 & 2.68 & 2.6 & 2.62 & 2.4 & 2.53 & 2.5 & 2.53 \\
\hline 59 & 2.57 & 2.64 & 2.62 & 2.63 & 2.54 & 2.59 & 2.66 & 2.62 & 2.45 & 2.61 & 2.52 & 2.66 & 2.6 & 2.6 & 2.58 & \\
\hline 60 & 2.55 & 2.62 & 2.57 & 2.57 & 2.66 & 2.6 & 2.71 & 2.51 & 2.71 & 2.58 & 2.67 & 2.72 & 2.72 & 2.57 & 2.69 & 2. \\
\hline 61 & 2.6 & 2.55 & 2.55 & 2.55 & 2.61 & 2.53 & 2.55 & 2.57 & 2.59 & 2.65 & 2.51 & 2.73 & 2.48 & 2.57 & 2.52 & 2.57 \\
\hline 62 & 2.7 & 2.63 & 2.72 & 2.72 & 2.58 & 2.8 & 2.64 & 2.71 & 2.74 & 2.74 & 2.66 & 2.62 & 2.68 & 2.53 & 2.51 & 2.55 \\
\hline 63 & 2.62 & 2.65 & 2.61 & 2.53 & 2.79 & 2.69 & 2.69 & 2.7 & 2.7 & 2.64 & 2.6 & 2.62 & 2.64 & 2.5 & 2.56 & 2.52 \\
\hline 64 & 2.62 & 2.62 & 2.54 & 2.75 & 2.59 & 2.58 & 2.6 & 2.61 & 2.61 & 2.54 & 2.57 & 2.65 & 2.53 & 2.57 & 2.53 & 2.64 \\
\hline 65 & 2.52 & 2.58 & 2.56 & 2.59 & 2.54 & 2.64 & 2.62 & 2.62 & 2.47 & 2.64 & 2.49 & 2.6 & 2.56 & 2.47 & 2.53 & 2.52 \\
\hline 66 & 2.53 & 2.6 & 2.57 & 2.77 & 2.51 & 2.54 & 2.51 & 2.55 & 2.45 & 2.74 & 2.53 & 2.62 & 2.55 & 2.5 & 2.53 & 2.61 \\
\hline 67 & 2.51 & 2.54 & 2.57 & 2.57 & 2.6 & 2.74 & 2.55 & 2.63 & 2.66 & 2.58 & 2.57 & 2.63 & 2.48 & 2.56 & 2.46 & 2.46 \\
\hline 68 & 2.63 & 2.54 & 2.6 & 2.52 & 2.59 & 2.6 & 2.68 & 2.5 & 2.64 & 2.53 & 2.66 & 2.57 & 2.57 & 2.6 & 2.65 & 2.51 \\
\hline 69 & 2.57 & 2.68 & 2.58 & 2.65 & 2.63 & 2.61 & 2.57 & 2.57 & 2.54 & 2.53 & 2.59 & 2.51 & 2.5 & 2.56 & 2.51 & 2.55 \\
\hline 70 & 2.55 & 2.56 & 2.58 & 2.66 & 2.78 & 2.63 & 2.69 & 2.7 & 2.71 & 2.63 & 2.71 & 2.6 & 2.62 & 2.63 & 2.63 & 2.56 \\
\hline 71 & 2.56 & 2.67 & 2.49 & 2.75 & 2.26 & 2.62 & 2.49 & 2.57 & 2.59 & 2.62 & 2.59 & 2.7 & 2.53 & 2.48 & 2.46 & 2.46 \\
\hline 72 & 2.74 & 2.51 & 2.61 & 2.55 & 2.75 & 2.58 & 2.63 & 2.6 & 2.74 & 2.6 & 2.6 & 2.56 & 2.65 & 2.51 & 2.65 & 2.55 \\
\hline 73 & 2.52 & 2.52 & 2.56 & 2.63 & 2.61 & 2.57 & 2.58 & 2.68 & 2.59 & 2.65 & 2.4 & 2.63 & 2.52 & 2.63 & 2.54 & 2.68 \\
\hline 74 & 2.53 & 2.47 & 2.55 & 2.55 & 2.5 & 2.64 & 2.51 & 2.57 & 2.59 & 2.55 & 2.64 & 2.51 & 2.44 & 2.44 & 2.57 & 2.55 \\
\hline 75 & 2.7 & 2.64 & 2.69 & 2.57 & 2.54 & 2.72 & 2.67 & 2.63 & 2.5 & 2.51 & 2.5 & 2.5 & 2.69 & 2.64 & 2.53 & 2.59 \\
\hline 76 & 2.32 & 2.46 & 2.5 & 2.59 & 2.42 & 2.54 & 2.46 & 2.63 & 2.48 & 2.58 & 2.54 & 2.54 & 2.48 & 2.57 & 2.43 & 2.66 \\
\hline 77 & 2.56 & 2.56 & 2.4 & 2.61 & 2.5 & 2.58 & 2.54 & 2.58 & 2.48 & 2.51 & 2.54 & 2.57 & 2.59 & 2.53 & 2.53 & 2.62 \\
\hline 78 & 2.63 & 2.48 & 2.62 & 2.56 & 2.48 & 2.53 & 2.51 & 2.5 & 2.59 & 2.59 & 2.56 & 2.61 & 2.62 & 2.56 & 2.58 & \\
\hline 79 & 2.45 & 2.48 & 2.58 & 2.52 & 2.52 & 2.59 & 2.52 & 2.61 & 2.51 & 2.55 & 2.55 & 2.64 & 2.45 & 2.56 & 2.52 & 2.61 \\
\hline 80 & 2.51 & 2.51 & 2.46 & 2.46 & 2.36 & 2.54 & 2.49 & 2.49 & 2.64 & 2.54 & 2.55 & 2.52 & 2.54 & 2.52 & 2.42 & 2.5 \\
\hline 81 & 2.62 & 2.72 & 2.58 & 2.63 & 2.72 & 2.72 & 2.59 & 2.62 & 2.6 & 2.51 & 2.6 & 2.53 & 2.65 & 2.58 & 2.5 & 2.62 \\
\hline 82 & 2.7 & 2.47 & 2.64 & 2.6 & 2.59 & 2.78 & 2.61 & 2.72 & 2.61 & 2.69 & 2.59 & 2.7 & 2.64 & 2.54 & 2.51 & 2.57 \\
\hline 83 & 2.56 & 2.63 & 2.61 & 2.67 & 2.63 & 2.6 & 2.69 & 2.62 & 2.59 & 2.69 & 2.57 & 2.57 & 2.57 & 2.62 & 2.63 & \\
\hline 84 & 2.58 & 2.62 & 2.58 & 2.61 & 2.7 & 2.61 & 2.48 & 2.59 & 2.59 & 2.52 & 2.57 & 2.46 & 2.52 & 2.57 & 2.57 & \\
\hline
\end{tabular}




\begin{tabular}{|c|c|c|c|c|c|c|c|c|c|c|c|c|c|c|c|c|}
\hline 85 & 2.59 & 2.6 & 2.6 & 2.62 & 2.57 & 2.57 & 2.61 & 2.63 & 2.57 & 2.57 & 2.83 & 2.58 & 2.55 & 2.54 & 2.59 & 2.59 \\
\hline 86 & 2.6 & 2.7 & 2.57 & 2.72 & 2.5 & 2.57 & 2.57 & 2.66 & 2.55 & 2.69 & 2.61 & 2.44 & 2.6 & 2.54 & 2.44 & 2.44 \\
\hline 87 & 2.67 & 2.47 & 2.63 & 2.48 & 2.69 & 2.63 & 2.64 & 2.6 & 2.57 & 2.52 & 2.62 & 2.55 & 2.55 & 2.5 & 2.55 & 2.53 \\
\hline 88 & 2.61 & 2.56 & 2.56 & 2.5 & 2.65 & 2.6 & 2.57 & 2.57 & 2.67 & 2.57 & 2.66 & 2.52 & 2.57 & 2.59 & 2.6 & 2.52 \\
\hline 89 & 2.69 & 2.6 & 2.76 & 2.65 & 2.63 & 2.64 & 2.56 & 2.69 & 2.65 & 2.56 & 2.62 & 2.65 & 2.54 & 2.6 & 2.58 & 2.63 \\
\hline 90 & 2.59 & 2.57 & 2.64 & 2.68 & 2.56 & 2.65 & 2.52 & 2.58 & 2.53 & 2.42 & 2.56 & 2.54 & 2.54 & 2.63 & 2.51 & 2.65 \\
\hline 91 & 2.63 & 2.51 & 2.52 & 2.62 & 2.61 & 2.55 & 2.61 & 2.59 & 2.69 & 2.54 & 2.66 & 2.54 & 2.58 & 2.62 & 2.64 & 2.31 \\
\hline 92 & 2.5 & 2.65 & 2.53 & 2.59 & 2.54 & 2.47 & 2.57 & 2.53 & 2.61 & 2.43 & 2.6 & 2.37 & 2.62 & 2.62 & 2.57 & \\
\hline 93 & 2.55 & 2.55 & 2.47 & 2.6 & 2.58 & 2.46 & 2.48 & 2.58 & 2.48 & 2.48 & 2.4 & 2.55 & 2.46 & 2.52 & 2.42 & 2.55 \\
\hline 94 & 2.53 & 2.59 & 2.51 & 2.63 & 2.6 & 2.48 & 2.65 & 2.56 & 2.56 & 2.61 & 2.64 & 2.56 & 2.58 & 2.49 & 2.58 & \\
\hline 95 & 2.6 & 2.66 & 2.66 & 2.7 & 2.56 & 2.5 & 2.59 & 2.5 & 2.54 & 2.56 & 2.55 & 2.54 & 2.51 & 2.58 & 2.52 & 2.61 \\
\hline 96 & 2.64 & 2.58 & 2.58 & 2.57 & 2.56 & 2.62 & 2.59 & 2.59 & 2.52 & 2.62 & 2.43 & 2.62 & 2.81 & 2.54 & 2.62 & \\
\hline 97 & 2.57 & 2.53 & 2.52 & 2.56 & 2.5 & 2.46 & 2.62 & 2.43 & 2.59 & 2.5 & 2.64 & 2.54 & 2.53 & 2.44 & 2.66 & \\
\hline 98 & 2.53 & 2.58 & 2.51 & 2.57 & 2.54 & 2.62 & 2.62 & 2.7 & 2.7 & 2.54 & 2.64 & 2.64 & 2.55 & 2.64 & 2.39 & 2.57 \\
\hline 99 & 2.6 & 2.57 & 2.58 & 2.56 & 2.57 & 2.62 & 2.55 & 2.6 & 2.65 & 2.59 & 2.66 & 2.61 & 2.63 & 2.53 & 2.55 & 2.52 \\
\hline 100 & 2.64 & 2.44 & 2.6 & 2.52 & 2.58 & 2.46 & 2.67 & 2.55 & 2.68 & 2.56 & 2.67 & 2.57 & 2.51 & 2.58 & 2.5 & 2.48 \\
\hline 101 & 2.57 & 2.54 & 2.55 & 2.51 & 2.43 & 2.68 & 2.42 & 2.61 & 2.61 & 2.51 & 2.61 & 2.64 & 2.54 & 2.54 & 2.54 & \\
\hline 102 & 2.57 & 2.6 & 2.55 & 2.56 & 2.67 & 2.65 & 2.62 & 2.68 & 2.57 & 2.59 & 2.53 & 2.5 & 2.59 & 2.59 & 2.72 & 2.56 \\
\hline 103 & 2.73 & 2.58 & 2.66 & 2.56 & 2.64 & 2.61 & 2.64 & 2.61 & 2.61 & 2.54 & 2.75 & 2.57 & 2.65 & 2.51 & 2.67 & 2.46 \\
\hline 104 & 2.55 & 2.7 & 2.66 & 2.57 & 2.57 & 2.59 & 2.52 & 2.62 & 2.69 & 2.49 & 2.58 & 2.53 & 2.53 & 2.58 & 2.46 & 2.5 \\
\hline 105 & 2.58 & 2.64 & 2.48 & 2.6 & 2.6 & 2.6 & 2.57 & 2.6 & 2.44 & 2.59 & 2.62 & 2.66 & 2.54 & 2.48 & 2.51 & 2.48 \\
\hline 106 & 2.49 & 2.54 & 2.52 & 2.6 & 2.58 & 2.59 & 2.58 & 2.56 & 2.52 & 2.62 & 2.53 & 2.57 & 2.45 & 2.54 & 2.48 & 2.61 \\
\hline 107 & 2.5 & 2.52 & 2.46 & 2.63 & 2.6 & 2.63 & 2.61 & 2.54 & 2.6 & 2.65 & 2.58 & 2.55 & 2.7 & 2.52 & 2.56 & \\
\hline 108 & 2.66 & 2.59 & 2.56 & 2.61 & 2.66 & 2.66 & 2.6 & 2.66 & 2.54 & 2.61 & 2.61 & 2.43 & 2.59 & 2.54 & 2.48 & 2.61 \\
\hline 109 & 2.59 & 2.44 & 2.5 & 2.43 & 2.49 & 2.8 & 2.52 & 2.65 & 2.48 & 2.54 & 2.52 & 2.59 & 2.41 & 2.52 & 2.55 & 2.62 \\
\hline 110 & 2.49 & 2.5 & 2.6 & 2.62 & 2.53 & 2.72 & 2.54 & 2.64 & 2.55 & 2.49 & 2.6 & 2.56 & 2.38 & 2.55 & 2.5 & 2.42 \\
\hline 111 & 2.59 & 2.59 & 2.52 & 2.52 & 2.58 & 2.55 & 2.63 & 2.59 & 2.61 & 2.61 & 2.61 & 2.61 & 2.52 & 2.61 & 2.43 & 2.5 \\
\hline 112 & 2.72 & 2.58 & 2.72 & 2.75 & 2.75 & 2.57 & 2.6 & 2.63 & 2.72 & 2.67 & 2.69 & 2.66 & 2.66 & 2.62 & 2.62 & 2.5 \\
\hline 113 & 2.44 & 2.64 & 2.51 & 2.59 & 2.65 & 2.64 & 2.61 & 2.56 & 2.55 & 2.59 & 2.64 & 2.61 & 2.44 & 2.48 & 2.45 & 2.64 \\
\hline 114 & 2.8 & 2.54 & 2.71 & 2.6 & 2.74 & 2.62 & 2.68 & 2.61 & 2.69 & 2.59 & 2.66 & 2.59 & 2.68 & 2.62 & 2.68 & 2.5 \\
\hline 115 & 2.73 & 2.67 & 2,81 & 2.6 & 2.66 & 2.69 & 2.59 & 2.62 & 2.65 & 2.51 & 2.58 & 2.54 & 2.67 & 2.49 & 2.61 & \\
\hline 116 & 2.48 & 2.66 & 2.56 & 2.59 & 2.59 & 2.59 & 2.48 & 2.56 & 2.56 & 2.56 & 2.58 & 2.62 & 2.56 & 2.56 & 2.52 & \\
\hline 117 & 2.57 & 2.57 & 2.51 & 2.55 & 2.66 & 2.69 & 2.67 & 2.7 & 2.55 & 2.57 & 2.57 & 2.49 & 2.52 & 2.55 & 2.46 & \\
\hline
\end{tabular}




\begin{tabular}{|c|c|c|c|c|c|c|c|c|c|c|c|c|c|c|c|c|}
\hline 118 & 2.56 & 2.62 & 2.48 & 2.63 & 2.64 & 2.5 & 2.62 & 2.55 & 2.61 & 2.62 & 2.59 & 2.59 & 2.59 & 2.51 & 2.43 & 2.5 \\
\hline 119 & 2.69 & 2.5 & 2.59 & 2.47 & 2.74 & 2.63 & 2.84 & 2.58 & 2.58 & 2.53 & 2.59 & 2.53 & 2.53 & 2.52 & 2.6 & 2.6 \\
\hline 120 & 2.55 & 2.53 & 2.53 & 2.52 & 2.52 & 2.61 & 2.51 & 2.49 & 2.53 & 2.57 & 2.53 & 2.53 & 2.69 & 2.65 & 2.61 & 2.65 \\
\hline 121 & 2.55 & 2.61 & 2.58 & 2.63 & 2.63 & 2.71 & 2.64 & 2.66 & 2.68 & 2.7 & 2.7 & 2.72 & 2.65 & 2.46 & 2.68 & 2.47 \\
\hline 122 & 2.51 & 2.51 & 2.58 & 2.54 & 2.56 & 2.58 & 2.58 & 2.53 & 2.59 & 2.59 & 2.64 & 2.58 & 2.54 & 2.7 & 2.54 & 2.59 \\
\hline 123 & 2.55 & 2.6 & 2.52 & 2.59 & 2.59 & 2.59 & 2.73 & 2.6 & 2.69 & 2.55 & 2.58 & 2.73 & 2.73 & 2.62 & 2.6 & 2.56 \\
\hline 124 & 2.6 & 2.56 & 2.56 & 2.63 & 2.63 & 2.65 & 2.63 & 2.68 & 2.68 & 2.68 & 2.77 & 2.71 & 2.6 & 2.47 & 2.66 & 2.48 \\
\hline 125 & 2.63 & 2.74 & 2.78 & 2.66 & 2.6 & 2.42 & 2.55 & 2.49 & 2.69 & 2.58 & 2.51 & 2.56 & 2.51 & 2.55 & 2.49 & 2.49 \\
\hline 126 & 2.43 & 2.55 & 2.5 & 2.53 & 2.57 & 2.56 & 2.49 & 2.59 & 2.4 & 2.66 & 2.5 & 2.61 & 2.58 & 2.52 & 2.63 & 2.66 \\
\hline 127 & 2.48 & 2.49 & 2.46 & 2.5 & 2.52 & 2.61 & 2.55 & 2.58 & 2.38 & 2.43 & 2.43 & 2.39 & 2.59 & 2.58 & 2.58 & 2.53 \\
\hline 128 & 2.6 & 2.6 & 2.49 & 2.6 & 2.52 & 2.64 & 2.52 & 2.55 & 2.55 & 2.54 & 2.49 & 2.69 & 2.42 & 2.5 & 2.58 & 2.59 \\
\hline 129 & 2.59 & 2.64 & 2.5 & 2.67 & 2.62 & 2.69 & 2.93 & 2.67 & 2.71 & 2.6 & 2.69 & 2.59 & 2.54 & 2.6 & 2.57 & 2.45 \\
\hline 130 & 2.49 & 2.52 & 2.53 & 2.66 & 2.59 & 2.52 & 2.6 & 2.47 & 2.66 & 2.61 & 2.57 & 2.65 & 2.53 & 2.59 & 2.5 & 2.61 \\
\hline 131 & 2.46 & 2.59 & 2.6 & 2.64 & 2.64 & 2.58 & 2.54 & 2.58 & 2.72 & 2.56 & 2.62 & 2.55 & 2.51 & 2.56 & 2.54 & 2.6 \\
\hline 132 & 2.67 & 2.59 & 2.73 & 2.58 & 2.56 & 2.65 & 2.58 & 2.64 & 2.57 & 2.57 & 2.54 & 2.65 & 2.67 & 2.59 & 2.61 & 2.68 \\
\hline 133 & 2.61 & 2.5 & 2.51 & 2.53 & 2.43 & 2.6 & 2.56 & 2.71 & 2.6 & 2.56 & 2.63 & 2.55 & 2.55 & 2.57 & 2.62 & 2.52 \\
\hline 134 & 2.66 & 2.7 & 2.64 & 2.6 & 2.54 & 2.54 & 2.48 & 2.5 & 2.56 & 2.56 & 2.56 & 2.57 & 2.62 & 2.58 & 2.54 & 2.56 \\
\hline 135 & \multicolumn{16}{|c|}{ BAD- crosstalk } \\
\hline 136 & 2.6 & 2.59 & 2.59 & 2.66 & 2.55 & 2.66 & 2.61 & 2.54 & 2.61 & 2.55 & 2.43 & 2.6 & 2.6 & 2.54 & 2.54 & 2.62 \\
\hline 137 & \multicolumn{16}{|c|}{ Channels $14,15,16$ bad } \\
\hline 138 & 2.62 & 2.71 & 2.58 & 2.67 & 2.67 & 2.55 & 2.68 & 2.49 & 2.63 & 2.44 & 2.66 & 2.6 & 2.48 & 2.58 & 2.44 & 2.59 \\
\hline 139 & 2.71 & 2.5 & 2.71 & 2.63 & 2.77 & 2.66 & 2.74 & 2.72 & 2.72 & 2.61 & 2.63 & 2.61 & 2.59 & 2.54 & 2.54 & 2.56 \\
\hline 140 & 2.59 & 2.55 & 2.68 & 2.52 & 2.74 & 2.55 & 2.67 & 2.58 & 2.54 & 2.58 & 2.67 & 2.56 & 2.73 & 2.53 & 2.71 & 2.62 \\
\hline 141 & 2.65 & 2.58 & 2.63 & 2.65 & 2.53 & 2.62 & 2.65 & 2.57 & 2.57 & 2.59 & 2.62 & 2.6 & 2.58 & 2.54 & 2.56 & 2.58 \\
\hline 142 & 2.72 & 2.47 & 2.63 & 2.71 & 2.59 & 2.52 & 2.53 & 2.61 & 2.51 & 2.58 & 2.49 & 2.56 & 2.54 & 2.53 & 2.54 & 2.53 \\
\hline 143 & \multicolumn{16}{|c|}{ No signal channel 9} \\
\hline 144 & \multicolumn{16}{|c|}{ Channels 5-8 bad } \\
\hline 145 & 2.69 & 2.48 & 2.6 & 2.58 & 2.64 & 2.56 & 2.61 & 2.4 & 2.57 & 2.55 & 2.4 & 2.53 & 2.61 & 2.55 & 2.64 & 2.5 \\
\hline 146 & 2.53 & 2.57 & 2.54 & 2.53 & 2.59 & 2.57 & 2.5 & 2.5 & 2.58 & 2.56 & 2.46 & 2.63 & 2.58 & 2.55 & 2.59 & 2.61 \\
\hline 147 & 2.54 & 2.45 & 2.59 & 2.51 & 2.58 & 2.57 & 2.54 & 2.6 & 2.6 & 2.53 & 2.49 & 2.71 & 2.65 & 2.54 & 2.51 & 2.48 \\
\hline 148 & 2.57 & 2.57 & 2.64 & 2.64 & 2.52 & 2.57 & 2.72 & 2.62 & 2.59 & 2.67 & 2.57 & 2.74 & 2.65 & 2.67 & 2.65 & 2.62 \\
\hline 149 & 2.6 & 2.65 & 2.6 & 2.6 & 2.6 & 2.61 & 2.61 & 2.63 & 2.58 & 2.54 & 2.65 & 2.65 & 2.71 & 2.75 & 2.53 & 2.76 \\
\hline 150 & 2.49 & 2.52 & 2.58 & 2.61 & 2.61 & 2.7 & 2.57 & 2.6 & 2.66 & 2.67 & 2.6 & 2.63 & 2.56 & 2.52 & 2.54 & 2.42 \\
\hline
\end{tabular}




\begin{tabular}{|c|c|c|c|c|c|c|c|c|c|c|c|c|c|c|c|c|}
\hline 151 & 2.54 & 2.65 & 2.5 & 2.57 & 2.4 & 2.49 & 2.56 & 2.56 & 2.55 & 2.61 & 2.44 & 2.51 & 2.41 & 2.54 & 2.48 & 2.52 \\
\hline 152 & 2.6 & 2.62 & 2.63 & 2.69 & 2.53 & 2.6 & 2.55 & 2.61 & 2.58 & 2.6 & 2.55 & 2.56 & 2.62 & 2.66 & 2.52 & 2.52 \\
\hline 153 & 2.57 & 2.46 & 2.56 & 2.53 & 2.41 & 2.5 & 2.6 & 2.51 & 2.61 & 2.52 & 2.61 & 2.51 & 2.51 & 2.57 & 2.55 & 2.64 \\
\hline 154 & 2.31 & 2.52 & 2.43 & 2.37 & 2.49 & 2.44 & 2.34 & 2.57 & 2.57 & 2.46 & 2.46 & 2.46 & 2.57 & 2.47 & 2.57 & 2.48 \\
\hline 155 & 2.63 & 2.5 & 2.56 & 2.68 & 2.58 & 2.64 & 2.61 & 2.58 & 2.55 & 2.53 & 2.62 & 2.62 & 2.62 & 2.39 & 2.53 & 2.54 \\
\hline 156 & 2.56 & 2.46 & 2.63 & 2.54 & 2.57 & 2.44 & 2.6 & 2.47 & 2.37 & 2.57 & 2.46 & 2.53 & 2.73 & 2.51 & 2.66 & 2.5 \\
\hline 157 & 2.64 & 2.52 & 2.66 & 2.58 & 2.52 & 2.49 & 2.54 & 2.54 & 2.58 & 2.63 & 2.63 & 2.55 & 2.49 & 2.69 & 2.46 & 2.65 \\
\hline 158 & 2.55 & 2.59 & 2.52 & 2.53 & 2.59 & 2.58 & 2.51 & 2.65 & 2.53 & 2.52 & 2.62 & 2.59 & 2.55 & 2.56 & 2.58 & 2.54 \\
\hline 159 & 2.68 & 2.56 & 2.64 & 2.64 & 2.64 & 2.64 & 2.64 & 2.59 & 2.71 & 2.52 & 2.62 & 2.59 & 2.68 & 2.73 & 2.6 & 2.6 \\
\hline 160 & 2.61 & 2.48 & 2.57 & 2.61 & 2.61 & 2.55 & 2.53 & 2.53 & 2.62 & 2.69 & 2.68 & 2.63 & 2.6 & 2.47 & 2.56 & 2.46 \\
\hline 161 & 2.54 & 2.46 & 2.65 & 2.58 & 2.56 & 2.55 & 2.46 & 2.53 & 2.5 & 2.5 & 2.55 & 2.51 & 2.44 & 2.41 & 2.49 & 2.63 \\
\hline 162 & 2.7 & 2.55 & 2.61 & 2.58 & 2.74 & 2.51 & 2.63 & 2.6 & 2.54 & 2.61 & 2.69 & 2.46 & 2.58 & 2.53 & 2.53 & 2.49 \\
\hline 163 & 2.65 & 2.6 & 2.5 & 2.63 & 2.51 & 2.54 & 2.54 & 2.59 & 2.51 & 2.62 & 2.5 & 2.59 & 2.59 & 2.59 & 2.51 & 2.56 \\
\hline 164 & 2.64 & 2.59 & 2.56 & 2.58 & 2.63 & 2.55 & 2.57 & 2.61 & 2.6 & 2.44 & 2.61 & 2.61 & 2.61 & 2.52 & 2.56 & 2.48 \\
\hline 165 & 2.66 & 2.41 & 2.62 & 2.51 & 2.61 & 2.46 & 2.55 & 2.46 & 2.65 & 2.67 & 2.68 & 2.5 & 2.51 & 2.61 & 2.53 & 2.61 \\
\hline 166 & 2.53 & 2.63 & 2.53 & 2.62 & 2.52 & 2.6 & 2.6 & 2.56 & 2.53 & 2.52 & 2.48 & 2.53 & 2.49 & 2.6 & 2.47 & 2.56 \\
\hline 167 & 2.54 & 2.68 & 2.56 & 2.6 & 2.55 & 2.65 & 2.53 & 2.64 & 2.57 & 2.63 & 2.65 & 2.58 & 2.63 & 2.51 & 2.7 & 2.54 \\
\hline 168 & $\begin{array}{l}2.52 \\
\text { BAD- } \\
\text { crossta }\end{array}$ & 2.52 & 2.62 & 2.54 & 2.6 & 2.53 & 2.63 & 2.52 & 2.63 & 2.58 & 2.56 & 2.54 & 2.6 & 2.49 & 2.57 & 2.52 \\
\hline 169 & $\mathrm{lk}$ & 2.55 & 2.51 & 2.64 & 2.66 & 2.7 & 2.64 & 2.67 & 2.58 & 2.67 & 2.58 & 2.61 & 2.45 & 2.58 & 2.45 & 2.65 \\
\hline 170 & 2.65 & 2.62 & 2.51 & 2.57 & 2.39 & 2.55 & 2.53 & 2.45 & 2.63 & 2.69 & 2.48 & 2.64 & 2.47 & 2.59 & 2.47 & 2.49 \\
\hline 171 & 2.6 & 2.6 & 2.57 & 2.62 & 2.46 & 2.55 & 2.47 & 2.56 & 2.61 & 2.55 & 2.53 & 2.58 & 2.49 & 2.51 & 2.46 & 2.56 \\
\hline 172 & 2.62 & 2.51 & 2.52 & 2.4 & 2.53 & 2.45 & 2.61 & 2.49 & 2.4 & 2.55 & 2.5 & 2.6 & 2.58 & 2.51 & 2.63 & 2.43 \\
\hline 173 & 2.3 & 2.3 & 2.29 & 2.23 & 2.3 & 2.28 & 2.29 & 2.28 & 2.28 & 2.38 & 2.22 & 2.31 & 2.31 & 2.21 & 2.21 & 2.27 \\
\hline 174 & 2.61 & 2.53 & 2.59 & 2.58 & 2.65 & 2.52 & 2.6 & 2.59 & 2.6 & 2.5 & 2.59 & 2.58 & 2.5 & 2.56 & 2.44 & 2.6 \\
\hline 175 & 2.5 & 2.55 & 2.5 & 2.48 & 2.52 & 2.6 & 2.49 & 2.48 & 2.5 & 2.48 & 2.48 & 2.54 & 2.55 & 2.65 & 2.58 & 2.5 \\
\hline 176 & 2.5 & 2.48 & 2.5 & 2.59 & 2.58 & 2.62 & 2.64 & 2.6 & 2.62 & 2.58 & 2.57 & 2.57 & 2.5 & 2.52 & 2.52 & 2.55 \\
\hline 177 & 2.64 & 2.55 & 2.56 & 2.64 & 2.72 & 2.6 & 2.68 & 2.53 & 2.64 & 2.59 & 2.62 & 2.43 & 2.53 & 2.57 & 2.57 & 2.61 \\
\hline 178 & 2.46 & 2.44 & 2.46 & 2.53 & 2.44 & 2.48 & 2.52 & 2.34 & 2.49 & 2.56 & 2.48 & 2.5 & 2.47 & 2.54 & 2.41 & 2.53 \\
\hline 179 & 2.55 & 2.61 & 2.52 & 2.57 & 2.6 & 2.58 & 2.55 & 2.56 & 2.55 & 2.53 & 2.53 & 2.54 & 2.55 & 2.55 & 2.52 & 2.64 \\
\hline 180 & 2.58 & 2.68 & 2.45 & 2.59 & 2.53 & 2.52 & 2.54 & 2.46 & 2.58 & 2.52 & 2.63 & 2.58 & 2.42 & 2.6 & 2.49 & 2.54 \\
\hline 181 & 2.56 & 2.61 & 2.6 & 2.54 & 2.6 & 2.44 & 2.6 & 2.46 & 2.51 & 2.43 & 2.61 & 2.59 & 2.65 & 2.6 & 2.55 & 2.52 \\
\hline
\end{tabular}




\begin{tabular}{|c|c|c|c|c|c|c|c|c|c|c|c|c|c|c|c|c|}
\hline 182 & 2.37 & 2.52 & 2.5 & 2.48 & 2.57 & 2.57 & 2.52 & 2.63 & 2.53 & 2.5 & 2.42 & 2.58 & 2.61 & 2.56 & 2.45 & 2.6 \\
\hline 183 & 2.68 & 2.57 & 2.58 & 2.53 & 2.55 & 2.49 & 2.57 & 2.58 & 2.73 & 2.6 & 2.62 & 2.59 & 2.5 & 2.55 & 2.52 & 2.53 \\
\hline 184 & 2.55 & 2.65 & 2.48 & 2.6 & 2.4 & 2.5 & 2.54 & 2.49 & 2.59 & 2.59 & 2.49 & 2.49 & 2.53 & 2.53 & 2.51 & 2.54 \\
\hline 185 & 2.66 & 2.59 & 2.62 & 2.62 & 2.58 & 2.56 & 2.52 & 2.57 & 2.55 & 2.51 & 2.45 & 2.8 & 2.55 & 2.55 & 2.5 & 2.54 \\
\hline 186 & 2.45 & 2.51 & 2.54 & 2.5 & 2.53 & 2.53 & 2.59 & 2.51 & 2.46 & 2.5 & 2.46 & 2.64 & 2.49 & 2.6 & 2.5 & 2.6 \\
\hline 187 & 2.53 & 2.58 & 2.57 & 2.57 & 2.53 & 2.68 & 2.51 & 2.55 & 2.48 & 2.48 & 2.55 & 2.44 & 2.52 & 2.56 & 2.43 & 2.55 \\
\hline 188 & 2.53 & 2.54 & 2.54 & 2.54 & 2.57 & 2.57 & 2.57 & 2.67 & 2.66 & 2.52 & 2.59 & 2.51 & 2.6 & 2.5 & 2.68 & 2.48 \\
\hline 189 & 2.48 & 2.66 & 2.5 & 2.7 & 2.52 & 2.54 & 2.76 & 2.42 & 2.58 & 2.45 & 2.61 & 2.54 & 2.46 & 2.6 & 2.5 & 2.6 \\
\hline
\end{tabular}

Card

\#

Crosstalk

Min.

int.

thresh

old Width

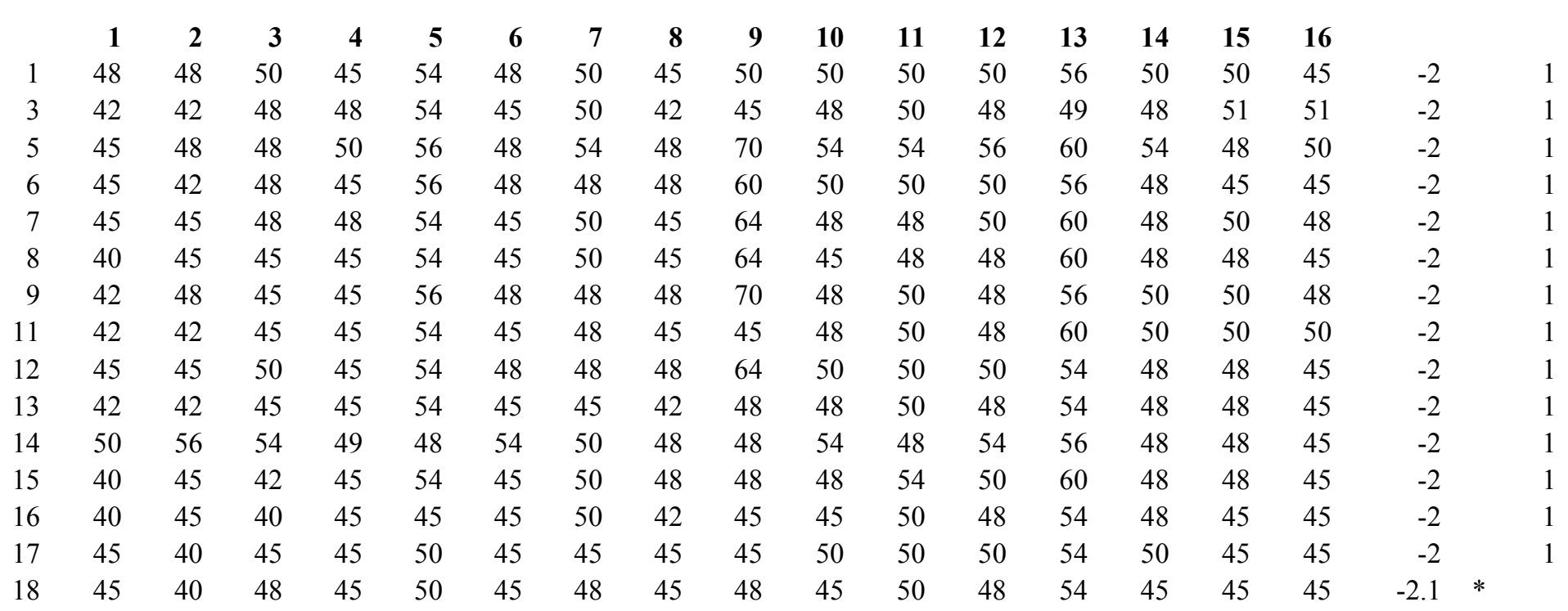




\begin{tabular}{|c|c|c|c|c|c|c|c|c|c|c|c|c|c|c|c|c|c|c|}
\hline 19 & 40 & 42 & 42 & 42 & 45 & 50 & 40 & 45 & 45 & 50 & 48 & 50 & 50 & 48 & 50 & 45 & -2 & \\
\hline 20 & 40 & 40 & 45 & 40 & 50 & 42 & 45 & 42 & 45 & 48 & 50 & 50 & 54 & 50 & 45 & 45 & -2 & \\
\hline 21 & 40 & 40 & 45 & 40 & 50 & 45 & 48 & 45 & 45 & 45 & 45 & 50 & 54 & 50 & 45 & 45 & -2 & \\
\hline 22 & 40 & 45 & 40 & 42 & 45 & 45 & 45 & 45 & 48 & 48 & 48 & 48 & 48 & 50 & 48 & 45 & -2.1 & \\
\hline 23 & 45 & 40 & 48 & 40 & 50 & 45 & 45 & 42 & 48 & 45 & 48 & 45 & 50 & 48 & 45 & 45 & -2 & \\
\hline 24 & 42 & 45 & 45 & 45 & 54 & 48 & 45 & 45 & 54 & 48 & 50 & 50 & 60 & 50 & 48 & 48 & -2 & \\
\hline 25 & 48 & 48 & 45 & 42 & 45 & 45 & 48 & 45 & 48 & 54 & 54 & 50 & 48 & 48 & 48 & 45 & -2.1 & \\
\hline 26 & 45 & 42 & 42 & 42 & 54 & 48 & 48 & 42 & 45 & 48 & 50 & 48 & 54 & 50 & 50 & 45 & -2 & \\
\hline 27 & 40 & 40 & 45 & 42 & 50 & 45 & 48 & 48 & 45 & 50 & 48 & 50 & 50 & 50 & 48 & 45 & -2.1 & \\
\hline 28 & 40 & 40 & 48 & 42 & 54 & 42 & 45 & 42 & 45 & 45 & 50 & 54 & 48 & 45 & 45 & 45 & -2 & \\
\hline 29 & 45 & 48 & 48 & 45 & 45 & 48 & 48 & 42 & 48 & 45 & 48 & 50 & 50 & 48 & 48 & 45 & -2 & \\
\hline 30 & 45 & 42 & 45 & 45 & 54 & 50 & 50 & 45 & 45 & 50 & 50 & 50 & 54 & 48 & 45 & 45 & -2 & \\
\hline 31 & 42 & 45 & 45 & 48 & 54 & 48 & 48 & 48 & 45 & 48 & 50 & 50 & 60 & 50 & 50 & 45 & -2.1 & \\
\hline 32 & 40 & 45 & 45 & 45 & 50 & 48 & 45 & 50 & 48 & 45 & 50 & 45 & 50 & 50 & 48 & 45 & -2 & \\
\hline 33 & 45 & 45 & 50 & 45 & 50 & 48 & 50 & 45 & 50 & 50 & 54 & 50 & 60 & 50 & 50 & 45 & -2 & \\
\hline 34 & 40 & 45 & 50 & 45 & 50 & 50 & 48 & 45 & 50 & 50 & 50 & 50 & 60 & 54 & 45 & 45 & -2.1 & \\
\hline 35 & 45 & 48 & 50 & 48 & 60 & 45 & 54 & 50 & 50 & 50 & 50 & 50 & 60 & 54 & 54 & 45 & -2 & \\
\hline 36 & 42 & 42 & 45 & 45 & 54 & 45 & 48 & 45 & 45 & 50 & 50 & 50 & 54 & 45 & 50 & 45 & -2 & \\
\hline 37 & 42 & 48 & 48 & 45 & 45 & 50 & 48 & 45 & 50 & 54 & 50 & 50 & 50 & 48 & 54 & 45 & -2 & $*$ \\
\hline 38 & 40 & 48 & 45 & 45 & 45 & 48 & 45 & 45 & 42 & 48 & 48 & 50 & 48 & 48 & 50 & 45 & -2 & \\
\hline 39 & 42 & 45 & 42 & 42 & 43 & 54 & 45 & 42 & 48 & 45 & 50 & 45 & 50 & 48 & 48 & 45 & -2.1 & \\
\hline 40 & 40 & 40 & 45 & 40 & 54 & 45 & 45 & 40 & 48 & 45 & 50 & 50 & 50 & 45 & 50 & 45 & -2 & \\
\hline 41 & 40 & 40 & 40 & 40 & 40 & 40 & 40 & 40 & 45 & 45 & 50 & 45 & 50 & 45 & 45 & 45 & -2.1 & \\
\hline 42 & \multicolumn{18}{|c|}{ first four channels, bad crosstalk } \\
\hline 43 & 45 & 40 & 45 & 45 & 50 & 45 & 50 & 45 & 45 & 50 & 50 & 50 & 54 & 48 & 45 & 48 & -2 & \\
\hline 44 & 40 & 45 & 45 & 45 & 60 & 45 & 50 & 50 & 45 & 50 & 45 & 50 & 54 & 50 & 45 & 45 & -2.2 & \\
\hline 45 & 40 & 45 & 45 & 45 & 45 & 50 & 45 & 45 & 45 & 50 & 45 & 50 & 50 & 50 & 55 & 40 & -2 & \\
\hline 46 & 40 & 42 & 45 & 40 & 54 & 45 & 50 & 45 & 50 & 48 & 50 & 50 & 54 & 50 & 45 & 45 & -2 & \\
\hline 47 & 45 & 40 & 45 & 40 & 45 & 45 & 45 & 45 & 45 & 50 & 50 & 50 & 54 & 48 & 50 & 45 & -2 & \\
\hline 48 & 45 & 48 & 48 & 45 & 54 & 45 & 45 & 45 & 45 & 50 & 50 & 50 & 54 & 45 & 50 & 50 & -2 & \\
\hline 49 & 45 & 45 & 45 & 45 & 54 & 45 & 45 & 45 & 48 & 50 & 54 & 50 & 54 & 50 & 45 & 45 & -2 & \\
\hline 50 & 40 & 45 & 45 & 42 & 45 & 50 & 50 & 45 & 48 & 48 & 45 & 50 & 45 & 45 & 48 & 45 & -2.2 & \\
\hline 51 & 48 & 45 & 50 & 45 & 54 & 48 & 50 & 45 & 48 & 45 & 48 & 48 & 54 & 45 & 45 & 60 & -1.9 & \\
\hline
\end{tabular}




\begin{tabular}{|c|c|c|c|c|c|c|c|c|c|c|c|c|c|c|c|c|c|}
\hline 52 & 40 & 48 & 45 & 45 & 45 & 45 & 40 & 42 & 45 & 48 & 45 & 50 & 45 & 45 & 45 & 48 & -2 \\
\hline 53 & 40 & 50 & 45 & 50 & 48 & 50 & 50 & 48 & 50 & 50 & 54 & 50 & 50 & 48 & 50 & 45 & -2 \\
\hline 54 & 42 & 45 & 50 & 45 & 54 & 50 & 50 & 48 & 50 & 54 & 50 & 54 & 54 & 50 & 50 & 50 & -2 \\
\hline 55 & 40 & 45 & 45 & 40 & 50 & 45 & 45 & 45 & 45 & 54 & 48 & 48 & 54 & 45 & 50 & 42 & -2.1 \\
\hline 56 & 45 & 45 & 50 & 45 & 54 & 48 & 50 & 48 & 50 & 50 & 50 & 50 & 60 & 50 & 50 & 45 & -2 \\
\hline 57 & 40 & 42 & 45 & 45 & 54 & 45 & 50 & 45 & 50 & 50 & 50 & 48 & 60 & 50 & 50 & 45 & -2 \\
\hline 58 & 48 & 45 & 50 & 45 & 54 & 48 & 54 & 50 & 45 & 48 & 50 & 50 & 60 & 50 & 50 & 48 & -2.1 \\
\hline 59 & 40 & 40 & 40 & 40 & 45 & 45 & 45 & 45 & 50 & 45 & 45 & 45 & 40 & 45 & 45 & 40 & -2 \\
\hline 60 & 45 & 45 & 45 & 45 & 50 & 45 & 45 & 45 & 45 & 50 & 45 & 45 & 50 & 50 & 45 & 45 & -2.1 \\
\hline 61 & 45 & 45 & 45 & 45 & 54 & 45 & 50 & 45 & 50 & 45 & 50 & 45 & 60 & 50 & 50 & 45 & 2 \\
\hline 62 & 40 & 45 & 40 & 40 & 45 & 40 & 40 & 40 & 40 & 45 & 45 & 45 & 45 & 45 & 45 & 40 & -2 \\
\hline 63 & 40 & 40 & 45 & 40 & 50 & 45 & 45 & 40 & 45 & 45 & 45 & 54 & 45 & 50 & 45 & 45 & -2.1 \\
\hline 64 & 45 & 40 & 50 & 40 & 54 & 45 & 50 & 45 & 45 & 50 & 50 & 54 & 54 & 50 & 50 & 45 & -2.1 \\
\hline 65 & 40 & 45 & 45 & 42 & 45 & 45 & 42 & 42 & 48 & 48 & 50 & 45 & 48 & 48 & 42 & 45 & -2.1 \\
\hline 66 & 45 & 45 & 50 & 45 & 54 & 50 & 50 & 45 & 50 & 48 & 50 & 50 & 60 & 50 & 50 & 45 & -2 \\
\hline 67 & 50 & 45 & 50 & 50 & 54 & 45 & 54 & 45 & 50 & 50 & 54 & 50 & 60 & 45 & 45 & 40 & -2 \\
\hline 68 & 40 & 48 & 42 & 45 & 45 & 45 & 42 & 45 & 45 & 50 & 45 & 48 & 48 & 45 & 45 & 45 & -2.1 \\
\hline 69 & 45 & 40 & 59 & 42 & 54 & 45 & 50 & 45 & 48 & 48 & 50 & 50 & 54 & 45 & 48 & 45 & -2 \\
\hline 70 & 40 & 45 & 45 & 40 & 40 & 45 & 40 & 40 & 45 & 45 & 45 & 45 & 45 & 45 & 45 & 40 & -1.9 \\
\hline 71 & 40 & 45 & 45 & 40 & 50 & 45 & 45 & 40 & 45 & 45 & 45 & 45 & 50 & 45 & 50 & 45 & -2.1 \\
\hline 72 & 40 & 48 & 45 & 45 & 42 & 48 & 42 & 45 & 42 & 48 & 48 & 50 & 48 & 48 & 45 & 45 & -2.1 \\
\hline 73 & 45 & 45 & 45 & 42 & 50 & 45 & 50 & 42 & 45 & 45 & 54 & 54 & 50 & 45 & 50 & 40 & -2 \\
\hline 74 & 40 & 45 & 40 & 40 & 54 & 45 & 48 & 40 & 40 & 45 & 45 & 50 & 54 & 45 & 45 & 45 & -2 \\
\hline 75 & 40 & 45 & 40 & 40 & 45 & 45 & 40 & 40 & 40 & 45 & 50 & 50 & 45 & 45 & 50 & 40 & -2.1 \\
\hline 76 & 50 & 48 & 50 & 45 & 54 & 50 & 54 & 45 & 50 & 48 & 54 & 50 & 60 & 50 & 54 & 45 & -2 \\
\hline 77 & 45 & 45 & 54 & 45 & 50 & 50 & 48 & 45 & 50 & 56 & 50 & 50 & 50 & 48 & 50 & 45 & -2.1 \\
\hline 78 & 42 & 49 & 45 & 54 & 48 & 48 & 45 & 45 & 45 & 48 & 50 & 54 & 45 & 48 & 48 & 45 & -2 \\
\hline 79 & 48 & 45 & 48 & 48 & 54 & 48 & 48 & 45 & 50 & 50 & 50 & 48 & 50 & 50 & 50 & 48 & -2 \\
\hline 80 & 45 & 48 & 50 & 48 & 56 & 48 & 48 & 48 & 48 & 48 & 54 & 50 & 56 & 50 & 50 & 45 & -2 \\
\hline 81 & 40 & 42 & 42 & 42 & 42 & 45 & 45 & 48 & 50 & 50 & 50 & 48 & 48 & 48 & 50 & 45 & -1.9 \\
\hline 82 & 40 & 45 & 45 & 45 & 54 & 48 & 48 & 40 & 48 & 45 & 50 & 48 & 54 & 50 & 48 & 45 & -2.1 \\
\hline 83 & 42 & 45 & 42 & 50 & 45 & 48 & 42 & 45 & 48 & 48 & 50 & 50 & 50 & 45 & 48 & 42 & -2.1 \\
\hline 84 & 45 & 42 & 48 & 45 & 50 & 45 & 50 & 45 & 48 & 50 & 50 & 54 & 54 & 48 & 48 & 45 & -2 \\
\hline
\end{tabular}




\begin{tabular}{|c|c|c|c|c|c|c|c|c|c|c|c|c|c|c|c|c|c|}
\hline 85 & 42 & 40 & 40 & 40 & 45 & 45 & 45 & 45 & 50 & 45 & 45 & 50 & 54 & 50 & 45 & 45 & -2.1 \\
\hline 86 & 40 & 40 & 42 & 40 & 50 & 42 & 45 & 40 & 45 & 45 & 45 & 50 & 50 & 45 & 48 & 40 & -1.9 \\
\hline 87 & 40 & 48 & 42 & 45 & 42 & 45 & 40 & 42 & 45 & 50 & 45 & 50 & 48 & 48 & 48 & 45 & -2.1 \\
\hline 88 & 40 & 40 & 45 & 45 & 50 & 45 & 48 & 45 & 45 & 48 & 45 & 50 & 50 & 45 & 45 & 45 & -2 \\
\hline 89 & 42 & 45 & 45 & 45 & 54 & 54 & 54 & 45 & 50 & 50 & 50 & 50 & 50 & 50 & 50 & 45 & -2 \\
\hline 90 & 40 & 40 & 50 & 45 & 54 & 50 & 50 & 45 & 48 & 54 & 50 & 50 & 54 & 45 & 48 & 45 & -2 \\
\hline 91 & 45 & 50 & 45 & 40 & 45 & 50 & 45 & 45 & 45 & 50 & 45 & 50 & 45 & 45 & 45 & 45 & -2 \\
\hline 92 & 45 & 45 & 45 & 45 & 45 & 50 & 45 & 45 & 45 & 50 & 50 & 54 & 54 & 48 & 45 & 45 & -2 \\
\hline 93 & 45 & 45 & 54 & 45 & 54 & 50 & 54 & 48 & 54 & 50 & 50 & 54 & 60 & 50 & 54 & 45 & -2.1 \\
\hline 94 & 40 & 45 & 45 & 42 & 45 & 50 & 42 & 45 & 48 & 48 & 48 & 48 & 48 & 48 & 45 & 45 & -2 \\
\hline 95 & 45 & 45 & 50 & 45 & 54 & 50 & 50 & 50 & 50 & 50 & 54 & 54 & 60 & 50 & 50 & 45 & -2 \\
\hline 96 & 42 & 48 & 45 & 45 & 45 & 45 & 45 & 45 & 48 & 48 & 50 & 50 & 50 & 50 & 45 & 48 & -2.1 \\
\hline 97 & 45 & 45 & 45 & 45 & 48 & 54 & 45 & 50 & 48 & 48 & 48 & 50 & 48 & 50 & 48 & 48 & -2 \\
\hline 98 & 45 & 40 & 50 & 45 & 54 & 45 & 50 & 48 & 45 & 48 & 50 & 50 & 60 & 45 & 50 & 45 & -1.9 \\
\hline 99 & 45 & 45 & 48 & 45 & 50 & 45 & 50 & 45 & 45 & 48 & 45 & 48 & 54 & 50 & 48 & 45 & -2 \\
\hline 100 & 45 & 48 & 48 & 48 & 54 & 50 & 50 & 48 & 50 & 50 & 50 & 50 & 60 & 48 & 50 & 50 & -2.1 \\
\hline 101 & 40 & 45 & 42 & 40 & 48 & 45 & 45 & 40 & 45 & 48 & 45 & 45 & 48 & 45 & 45 & 40 & -2 \\
\hline 102 & 40 & 45 & 42 & 42 & 42 & 45 & 42 & 40 & 45 & 45 & 48 & 48 & 48 & 45 & 40 & 42 & -2 \\
\hline 103 & 40 & 45 & 45 & 45 & 50 & 45 & 50 & 45 & 45 & 50 & 48 & 50 & 54 & 50 & 45 & 50 & -2.2 \\
\hline 104 & 45 & 40 & 45 & 45 & 50 & 45 & 50 & 42 & 45 & 50 & 50 & 50 & 54 & 50 & 50 & 45 & -2.1 \\
\hline 105 & 40 & 45 & 50 & 45 & 54 & 45 & 50 & 45 & 50 & 50 & 50 & 50 & 60 & 54 & 50 & 45 & -2.1 \\
\hline 106 & 42 & 42 & 45 & 42 & 45 & 48 & 42 & 42 & 48 & 45 & 48 & 48 & 50 & 48 & 50 & 42 & -2.1 \\
\hline 107 & 45 & 45 & 50 & 45 & 54 & 45 & 50 & 45 & 45 & 45 & 50 & 50 & 50 & 48 & 45 & 48 & -2 \\
\hline 108 & 40 & 40 & 45 & 45 & 50 & 42 & 45 & 45 & 48 & 48 & 50 & 54 & 50 & 50 & 50 & 45 & -1.9 \\
\hline 109 & 45 & 45 & 48 & 48 & 54 & 40 & 50 & 45 & 50 & 50 & 50 & 50 & 60 & 50 & 45 & 45 & -2 \\
\hline 110 & 45 & 42 & 45 & 45 & 54 & 42 & 48 & 42 & 45 & 45 & 50 & 45 & 60 & 50 & 48 & 45 & -1.9 \\
\hline 111 & 42 & 45 & 45 & 45 & 45 & 50 & 45 & 45 & 50 & 50 & 50 & 50 & 50 & 45 & 54 & 45 & -2 \\
\hline 112 & 45 & 50 & 45 & 45 & 45 & 54 & 50 & 50 & 45 & 50 & 50 & 50 & 45 & 45 & 50 & 45 & -1.9 \\
\hline 113 & 45 & 45 & 45 & 45 & 45 & 45 & 45 & 45 & 50 & 50 & 48 & 54 & 50 & 54 & 54 & 45 & -2.1 \\
\hline 114 & 40 & 45 & 45 & 45 & 45 & 45 & 45 & 45 & 45 & 45 & 48 & 50 & 50 & 45 & 45 & 42 & -2.2 \\
\hline 115 & 40 & 45 & 45 & 45 & 50 & 45 & 50 & 45 & 45 & 80 & 50 & 50 & 50 & 50 & 48 & 48 & -2 \\
\hline 116 & 48 & 45 & 50 & 45 & 54 & 45 & 50 & 45 & 50 & 50 & 50 & 50 & 50 & 50 & 50 & 45 & -2 \\
\hline 117 & 45 & 42 & 48 & 45 & 50 & 42 & 45 & 40 & 48 & 50 & 50 & 54 & 54 & 50 & 50 & 45 & -1.9 \\
\hline
\end{tabular}




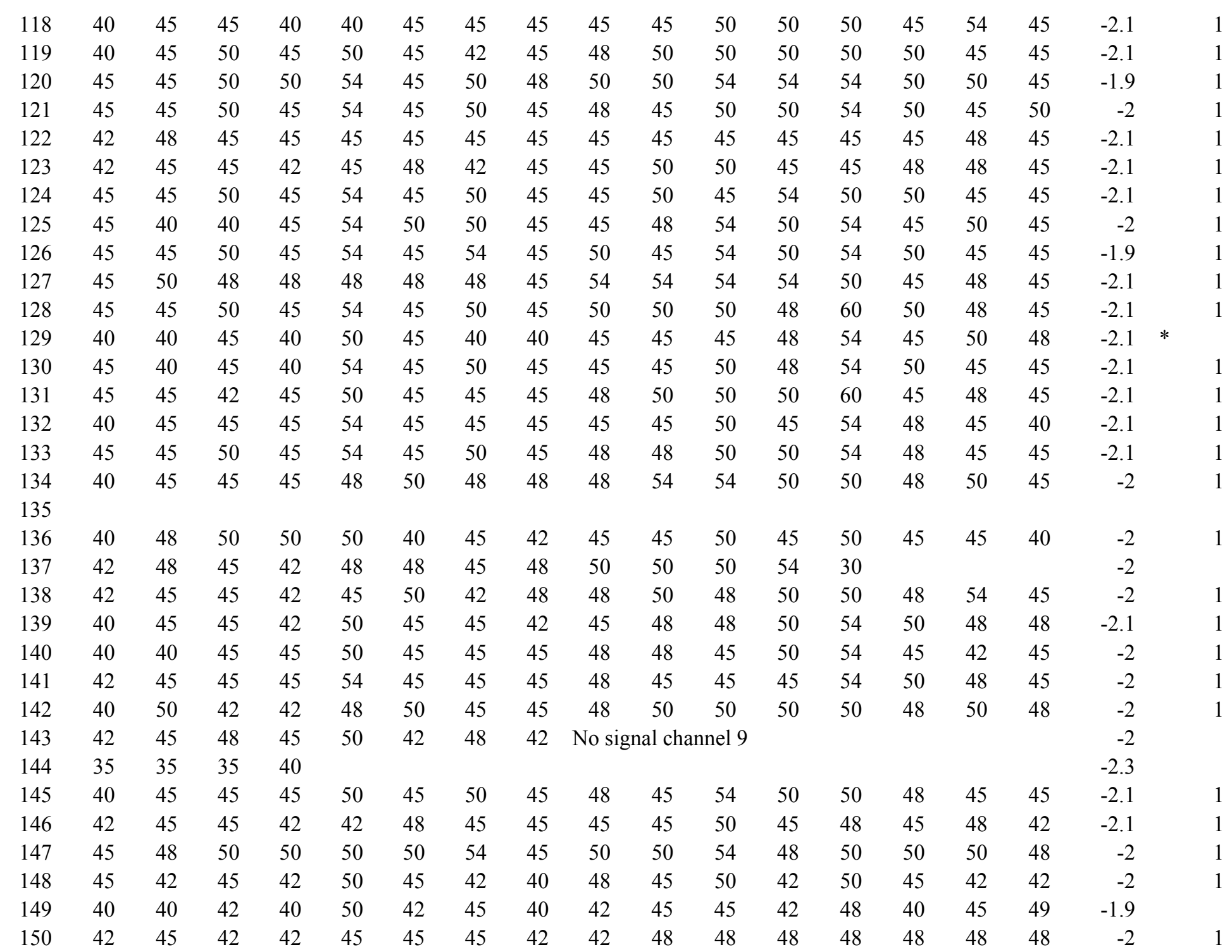




\begin{tabular}{|c|c|c|c|c|c|c|c|c|c|c|c|c|c|c|c|c|c|c|}
\hline 151 & 42 & 45 & 48 & 45 & 50 & 50 & 45 & 42 & 48 & 48 & 54 & 50 & 54 & 48 & 50 & 48 & -2 & 1 \\
\hline 152 & 40 & 40 & 45 & 40 & 50 & 42 & 50 & 42 & 45 & 45 & 50 & 45 & 54 & 40 & 45 & 42 & -1.8 & \\
\hline 153 & 45 & 48 & 50 & 45 & 54 & 50 & 50 & 48 & 50 & 50 & 50 & 54 & 54 & 50 & 50 & 48 & -2.1 & 1 \\
\hline 154 & 48 & 48 & 48 & 48 & 54 & 48 & 50 & 42 & 60 & 48 & 50 & 50 & 54 & 48 & 45 & 45 & -2 & 1 \\
\hline 155 & 40 & 42 & 45 & 40 & 54 & 42 & 49 & 42 & 63 & 50 & 48 & 48 & 54 & 50 & 45 & 45 & -2 & \\
\hline 156 & 40 & 40 & 40 & 40 & 50 & 45 & 45 & 45 & 50 & 45 & 50 & 45 & 50 & 45 & 42 & 45 & -2 & 1 \\
\hline 157 & 45 & 48 & 48 & 48 & 54 & 48 & 50 & 48 & 48 & 48 & 50 & 50 & 60 & 48 & 54 & 48 & -2 & 1 \\
\hline 158 & 42 & 42 & 48 & 45 & 50 & 45 & 48 & 42 & 45 & 45 & 45 & 48 & 54 & 45 & 45 & 45 & -2 & 1 \\
\hline 159 & 40 & 45 & 50 & 45 & 60 & 48 & 50 & 45 & 48 & 54 & 54 & 50 & 60 & 50 & 45 & 50 & -2 & 1 \\
\hline 160 & 50 & 45 & 45 & 42 & 48 & 45 & 48 & 45 & 42 & 42 & 42 & 45 & 50 & 48 & 45 & 45 & -2 & 1 \\
\hline 161 & 45 & 48 & 48 & 48 & 54 & 50 & 54 & 50 & 50 & 50 & 50 & 54 & 60 & 54 & 50 & 48 & -2 & 1 \\
\hline 162 & 40 & 42 & 45 & 42 & 45 & 45 & 45 & 42 & 45 & 45 & 42 & 50 & 54 & 48 & 48 & 45 & -2.1 & 1 \\
\hline 163 & 32 & 42 & 50 & 50 & 54 & 45 & 50 & 45 & 50 & 45 & 50 & 50 & 54 & 48 & 48 & 45 & 2 & 1 \\
\hline 164 & 42 & 40 & 48 & 45 & 54 & 45 & 48 & 45 & 48 & 50 & 48 & 50 & 54 & 48 & 48 & 48 & -2 & 1 \\
\hline 165 & 42 & 48 & 45 & 45 & 50 & 48 & 45 & 45 & 48 & 45 & 48 & 54 & 54 & 48 & 50 & 45 & -2.1 & 1 \\
\hline 166 & 45 & 40 & 48 & 42 & 54 & 45 & 45 & 45 & 50 & 45 & 50 & 50 & 60 & 48 & 50 & 45 & -2 & 1 \\
\hline 167 & 42 & 40 & 45 & 42 & 54 & 45 & 48 & 42 & 45 & 45 & 45 & 48 & 54 & 48 & 45 & 45 & -2.2 & 1 \\
\hline 168 & 42 & 45 & 42 & 45 & 50 & 45 & 45 & 42 & 45 & 45 & 50 & 50 & 60 & 50 & 48 & 45 & -2 & 1 \\
\hline 169 & \multicolumn{17}{|c|}{ crosstalk from channel 2 , card set aside } & \\
\hline 170 & 40 & 40 & 45 & 42 & 54 & 45 & 45 & 45 & 45 & 45 & 50 & 45 & 54 & 45 & 45 & 45 & -2.1 & 1 \\
\hline 171 & 45 & 42 & 48 & 45 & 54 & 45 & 50 & 45 & 45 & 50 & 50 & 50 & 54 & 50 & 48 & 45 & -2 & 1 \\
\hline 172 & 40 & 45 & 45 & 45 & 45 & 45 & 45 & 45 & 48 & 45 & 50 & 45 & 45 & 45 & 45 & 45 & -2 & 1 \\
\hline 173 & 42 & 42 & 45 & 45 & 54 & 45 & 48 & 45 & 48 & 45 & 50 & 48 & 54 & 50 & 50 & 42 & -2 & 1 \\
\hline 174 & 42 & 45 & 48 & 45 & 45 & 48 & 48 & 45 & 45 & 50 & 50 & 50 & 50 & 48 & 50 & 45 & -2 & 1 \\
\hline 175 & 42 & 45 & 48 & 45 & 48 & 45 & 48 & 45 & 48 & 45 & 48 & 48 & 50 & 48 & 50 & 45 & -2 & 1 \\
\hline 176 & 45 & 45 & 50 & 45 & 45 & 48 & 45 & 45 & 45 & 48 & 48 & 48 & 50 & 48 & 50 & 45 & -2 & 1 \\
\hline 177 & 40 & 45 & 48 & 42 & 48 & 45 & 45 & 45 & 45 & 48 & 48 & 54 & 54 & 48 & 48 & 45 & -2.2 & 1 \\
\hline 178 & 45 & 48 & 48 & 54 & 48 & 50 & 45 & 48 & 48 & 48 & 50 & 56 & 50 & 48 & 50 & 45 & -2 & 1 \\
\hline 179 & 42 & 45 & 45 & 45 & 45 & 50 & 45 & 45 & 48 & 50 & 50 & 50 & 50 & 54 & 50 & 45 & -2 & 1 \\
\hline 180 & 42 & 45 & 48 & 42 & 48 & 50 & 45 & 45 & 45 & 50 & 45 & 50 & 56 & 48 & 48 & 45 & -2 & 1 \\
\hline 181 & 42 & 42 & 45 & 45 & 45 & 50 & 42 & 45 & 48 & 54 & 48 & 48 & 45 & 48 & 48 & 45 & -2.1 & 1 \\
\hline 182 & 48 & 45 & 50 & 48 & 48 & 50 & 48 & 45 & 50 & 54 & 54 & 50 & 50 & 48 & 54 & 45 & -2 & 1 \\
\hline 183 & 40 & 40 & 45 & 42 & 50 & 45 & 45 & 42 & 48 & 45 & 48 & 48 & 54 & 45 & 48 & 45 & -2 & 1 \\
\hline
\end{tabular}




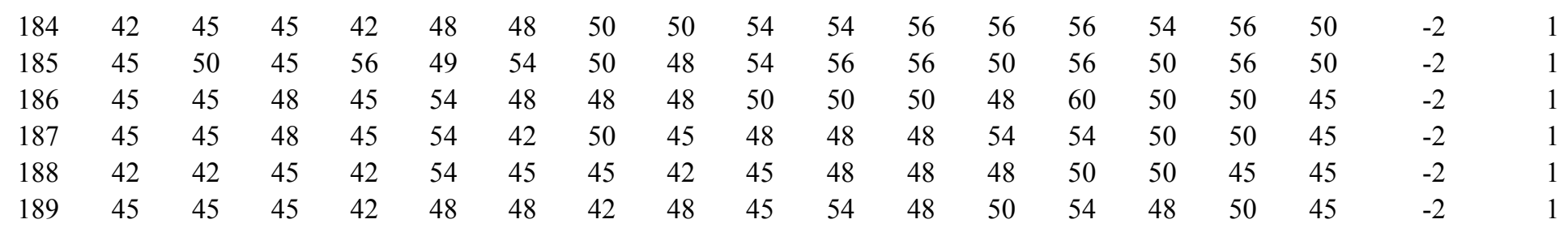


